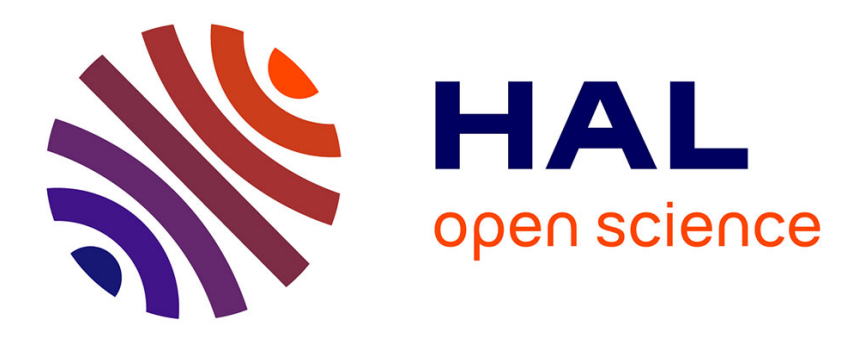

\title{
Observing and Controlling the Folding Pathway of DNA Origami at the Nanoscale
}

\author{
Jonathan Lee Tin Wah, Christophe David, Sergii Rudiuk, Damien Baigl, \\ André Estévez-Torres
}

\section{- To cite this version:}

Jonathan Lee Tin Wah, Christophe David, Sergii Rudiuk, Damien Baigl, André Estévez-Torres. Observing and Controlling the Folding Pathway of DNA Origami at the Nanoscale. ACS Nano, 2016, 10 (2), pp.1978 - 1987. 10.1021/acsnano.5b05972 . hal-01623551

\section{HAL Id: hal-01623551 https: / hal.sorbonne-universite.fr/hal-01623551}

Submitted on 25 Oct 2017

HAL is a multi-disciplinary open access archive for the deposit and dissemination of scientific research documents, whether they are published or not. The documents may come from teaching and research institutions in France or abroad, or from public or private research centers.
L'archive ouverte pluridisciplinaire HAL, est destinée au dépôt et à la diffusion de documents scientifiques de niveau recherche, publiés ou non, émanant des établissements d'enseignement et de recherche français ou étrangers, des laboratoires publics ou privés. 


\section{Observing and Controlling the Folding Pathway of DNA Origami at the Nanoscale}

Jonathan Lee Tin Wah, ${ }^{\dagger \neq}$ Christophe David, " Sergii Rudiuk, ${ }^{\S, \|, \perp}$ Damien

$$
\text { Baigl }, \S, \|, \perp \text { and André Estevez-Torres } *, \dagger, \ddagger
$$

Université Pierre et Marie Curie, Laboratoire Jean Perrin, 4 place Jussieu, 75005 Paris, France, CNRS, UMR 8237, Laboratoire de photonique et de nanostructures, CNRS, route de Nozay, 91460 Marcoussis, France, Ecole Normale Supérieure-PSL Research University, Department of Chemistry, 24 Rue Lhomond, 75005, Paris, France, Sorbonne Universités, UPMC Univ Paris 06, PASTEUR, 75005, Paris, France, and CNRS, UMR 8640 PASTEUR, 75005, Paris, France

E-mail: andre.estevez-torres@upmc.fr

${ }^{*}$ To whom correspondence should be addressed

${ }^{\dagger}$ Université Pierre et Marie Curie, Laboratoire Jean Perrin, 4 place Jussieu, 75005 Paris, France ${ }_{\ddagger}^{\ddagger} \mathrm{CNRS}, \mathrm{UMR} 8237$

\Laboratoire de photonique et de nanostructures, CNRS, route de Nozay, 91460 Marcoussis, France

$\S$ Ecole Normale Supérieure-PSL Research University, Department of Chemistry, 24 Rue Lhomond, 75005, Paris, France

"Sorbonne Universités, UPMC Univ Paris 06, PASTEUR, 75005, Paris, France

${ }^{\perp}$ CNRS, UMR 8640 PASTEUR, 75005, Paris, France 


\begin{abstract}
DNA origami is a powerful method to fold DNA into rationally designed nanostructures that holds great promise for bio-nanotechnology. However, the folding mechanism has yet to be fully resolved, principally due to a lack of data with single molecule resolution. To address this issue, we have investigated in detail, using atomic force microscopy, the morphological evolution of hundreds of individual rectangular origamis in solution as a function of temperature. Significant structural changes were observed between 65 and $55{ }^{\circ} \mathrm{C}$ both for folding and melting and six structural intermediates were identified. In standard conditions folding was initiated at the edges of the rectangle and progressed towards the center. Melting happened through the reverse pathway until the structures were significantly disrupted but ended through a different pathway involving out-of-equilibrium chain-like structures. Increasing the relative concentration of center to edge staples dramatically modified the folding pathway to a mechanism progressing from the center towards the edges. These results indicate that the folding pathway is determined by thermodynamics and suggest a way of controlling it.
\end{abstract}

\title{
Keywords
}

DNA nanotechnology, AFM, image processing, nanostructure 
Structural DNA nanotechnology designs nanostructures using DNA strands as building blocks. ${ }^{1-5}$ In contrast with standard top-down fabrication techniques, such as lithography, it is a bottom-up process: billions of DNA strands are mixed in a water solution and selfassemble into the final nanostructures. DNA building blocks are particularly advantageous because the self-assembly rules are simple and relatively robust, with Watson-Crick pairing as the principal rule.

A small revolution happened in the field when P. W. Rothemund invented DNA origami: ${ }^{3}$ mixing a 7249 bases-long single stranded DNA (ssDNA), the scaffold, with a set of welldesigned oligonucleotides, about 32 bases long, the staples, allowed the fabrication of arbitrary two-dimensional shapes about 100-nm across. Later, three-dimensional structures could also be obtained following a similar principle. ${ }^{6-8}$ Recently, Yin and collaborators introduced single stranded tile assembly, where arbitrary 2D and 3D structures were selfassembled from oligonucleotides alone. ${ }^{9}$ Hollow 3D structures were also obtained by designing new scaffolds using surface rendering principles. ${ }^{10}$

Since the advent of DNA origami almost a decade ago the progress of DNA nanotechnology has been enormous as attested by the incipient applications of this technique, ${ }^{11}$ for example, for single molecule force measurements, ${ }^{12}$ fabrication of artificial nanopores ${ }^{13}$ and plasmonics. ${ }^{14}$ However, few studies have investigated the folding process or attempted to control it. The melting of a DNA origami rectangle adsorbed on mica was studied using a temperature-controlled AFM. ${ }^{15}$ This seminal work posed the problem for the first time, although it did not characterize the melting of DNA origami in solution but the thermodynamics of DNA origami bound to mica. The strong electrostatic forces between these two objects suggest that one should be cautious when extending these results to DNA origami in free solution. ${ }^{16}$ With a temperature-jump method involving a fluorescent DNA binder as read-out, Dietz and co-workers determined that 3D origami folding rate was heavily dependent on the temperature. ${ }^{17}$ This work allowed, for instance, to reduce the folding time of $3 \mathrm{D}$ origami from 1 week $^{6}$ to $2 \mathrm{~h},{ }^{17}$ indicating that fundamental thermodynamic studies may 
have a major influence in technological processes. Recently, insight about the thermodynamics of folding/melting of 2D and 3D origamis was provided by studying the melting curves of DNA origami in bulk using the FRET signal from two fluorescently-labeled staples. ${ }^{9,18}$ However, none of the aforementioned works has characterized the folding of DNA origami in solution with single-molecule resolution. Such level of detail is needed for building good theoretical models, that are currently scarce. ${ }^{19}$ Recently, a double scaffold design was used to infer folding pathways from the AFM images of the folded state alone ${ }^{20}$ and single-molecule force spectroscopy was employed to investigate and control folding. ${ }^{21}$

Here, inspired from these seminal works, we used AFM to take snapshots of the folding and melting of DNA origami structures in solution during a temperature ramp. We show evidence of at least six different folding states, with folding nucleation happening at the upper and lower edges of the tall rectangular structure investigated here. As temperature decreases, the nucleation sites grow towards the center of the structure, and finally merge to form the fully folded rectangle. A dedicated image analysis procedure allows us to extract a wealth of quantitative data from AFM images. Moreover, we show that the folding pathway can be dramatically modified by changing the concentration of a portion of the staples. Eventually, we demonstrate that data from AFM are consistent with experiments in bulk.

\section{Results and discussion}

For all the experiments we used the tall rectangle origami designed by Rothemund ${ }^{3}$ as it was previously studied thermodynamically. ${ }^{15}$ To get insight about the folding process at the single-molecule level we set up a protocol to take AFM snapshots of individual origami particles at different temperatures (Figure 1). To do so, aliquots of a 1:52 nM scaffold:staple solution were heated at $70{ }^{\circ} \mathrm{C}$ and then cooled at a rate of $1{ }^{\circ} \mathrm{C} / 15 \mathrm{~min}$. For every temperature, $T_{i}$, a piece of mica was immersed in the solution for 1 min to let the particles adsorb on the surface. Subsequently, the mica was washed in pure buffer at $T_{i}$. The excess of salt 
was removed by rapidly rinsing with water at room temperature. The sample was finally dried and imaged by AFM at room temperature. This method has several advantages. First, the folding process occurs in solution, without the presence of the mica surface. Only when folding has reached thermodynamic equilibrium is the solution put in contact with the mica. As a result, adsorption is not expected to change significantly the folding pathway. Second, sample drying enables us to 'freeze' the origami in its equilibrium structure by stopping both the melting of formed structures - that could occur if the mica was imaged in pure bufferand the hybridization of staples contained in the starting solution.

\section{Folding proceeds through five distinct intermediates some of them coexististing at a given temperature}

First, we used this adsorption/drying method to study the folding process. Figure 2 displays AFM images of single foldamers at different temperatures during cooling. One can visually distinguish at least six distinct states: linear (at $70-69{ }^{\circ} \mathrm{C}$, dark blue arrows), entangled coil $\left(70-63{ }^{\circ} \mathrm{C}\right.$, pink arrows), half-width folded $\left(65-63{ }^{\circ} \mathrm{C}\right.$, green arrows), full-width folded $\left(63-61{ }^{\circ} \mathrm{C}\right.$, red arrows), broken rectangles $\left(63-55^{\circ} \mathrm{C}\right.$, orange arrows) and fully folded structures $\left(59-40{ }^{\circ} \mathrm{C}\right.$, light blue arrows). Several of these structures coexisted at a given temperature and the fraction of structures that are closer to the folded state increased, as expected, when temperature decreased.

Linear structures were typically $0.4 \pm 0.2 \mathrm{~nm}$ height and $9 \mathrm{~nm}$ in width with lengths up to $960 \mathrm{~nm}$, that we interpret as ssDNA portions of the M13 scaffold that have been stretched during sample drying, ${ }^{22}$ and probably do not exist in solution. However, they indicate coil configurations that are less strongly held together in solution than entangled coils. Entangled coils had typical dimensions of $150 \times 90 \mathrm{~nm}^{2}$ and were $0.5 \pm 0.2 \mathrm{~nm}$ high, which is compatible with ssDNA. The first clear structuration was observed at $65{ }^{\circ} \mathrm{C}$ : small rectangular portions that may reach $36 \times 15 \mathrm{~nm}^{2}$ across and $1.2 \mathrm{~nm}$ high were identified with half-width folded portions of the origami (from the border to the central seam). As temperature decreased 
the number of large rectangles increased, as well as their size, $72 \times 24 \mathrm{~nm}^{2}$ at $63{ }^{\circ} \mathrm{C}$ and $72 \times 34 \mathrm{~nm}^{2}$ at $61^{\circ} \mathrm{C}$. We identify them to full-width folded portions of the upper or lower part of the rectangle running from the left to the right border across the central seam. Among them, we distinguished either full-width folded and broken rectangle structures, where two full-width folded portions were held together by a short unstructured region. At $59{ }^{\circ} \mathrm{C}$, a majority of particles displayed a broken rectangle structure and at least one was almost perfectly folded. At 57 and $55{ }^{\circ} \mathrm{C}$ there was a mixture of broken and fully folded rectangles, the former being majority at 57 while the later dominating at $55{ }^{\circ} \mathrm{C}$ (SI Table S1). Between 50 and $40{ }^{\circ} \mathrm{C}$, images were practically indistinguishable (SI Figure S1 and Figure 2) and no partially folded structures were observed. At these temperatures, $85 \%$ of the particles folded without defects, $5 \%$ displayed large holes up to $1 / 2$ of the tall rectangle surface, and $10 \%$ were rectangular particles smaller than $1 / 4$ of the surface of the rectangle.

The full-width folded and broken rectangle structures are particularly interesting. The folded structures appeared always on the top and bottom ends of the tall rectangle, and not in the middle, indicating that folding is not random but starts at well-defined positions of the structure. This result is in agreement with the simulations of Song et al. ${ }^{15}$ who reported that the calculated melting temperatures of the staples are significantly higher on the top and bottom ends of the tall rectangle. Moreover, their simulation predicted a region of $24 \times 35 \mathrm{~nm}^{2}$ on the bottom left of the rectangle with particularly high $\left(>63{ }^{\circ} \mathrm{C}\right)$ melting temperature. This region is compatible with the half-width folded structures observed here at 65 and $63^{\circ} \mathrm{C}$. Our results are also in agreement with the recent study of Dunn et al. ${ }^{20}$ that confirmed that DNA origami folding is highly cooperative and found that early connections (due to staples with high melting temperatures) strongly influenced the folding pathway.

Our data show that little folding happened above $63{ }^{\circ} \mathrm{C}$, suggesting that these temperatures are not necessary for correct folding. To test this, we heated an origami solution at $95{ }^{\circ} \mathrm{C}$, and we followed the same cooling ramp as in Figure 2 except that we skipped the initial part by cooling the sample rapidly to temperature $T_{i}$ at $1^{\circ} \mathrm{C} / 0.2 \mathrm{~s}$ prior to normal 
cooling at $1^{\circ} \mathrm{C} / 15$ min down to $40{ }^{\circ} \mathrm{C}$. Interestingly, we obtained well-formed rectangles down to $T_{i}=57^{\circ} \mathrm{C}$ (SI Figure S10), a first indication that the folding process is under thermodynamic rather than kinetic control. In a more practical perspective, it shows that our method can be used to identify the temperature where critical folding events occur and therefore significantly simplify the origami formation protocol by focusing the cooling procedure to the range of temperatures where folding takes place. ${ }^{17}$

\section{Quantitative analysis of the folding process}

Second, to gain quantitative insight into the folding process, we used a segmentation algorithm to detect and analyze individual particles in the AFM images (see Methods below). Our segmentation procedure succeeded to detect individual particles at $59{ }^{\circ} \mathrm{C}$ and below (SI Figure S1). Above this temperature, the density of adsorbed particles was too large for distinguishing individual structures (SI Figure S2) and lowering it would result in poor sampling statistics at lower temperatures. To quantify the structural changes of these particles during folding we chose three parameters: the maximum end-to-end size and the modal and the median height (Figure 3A). Note that both heights were calculated by taking into account all pixels within the perimeter of the particle and thus holes were counted (Figure 1). Histograms of the end-to-end size (in the following noted size) displayed five distinct shapes, which is consistent with the six structures detected by visual inspection. As one may expect, the modal size and the width of the distribution decreased during cooling. The modal size decreased from $210 \pm 10 \mathrm{~nm}$ at high temperature until it stabilized at $140 \pm 10 \mathrm{~nm}$ at $57^{\circ} \mathrm{C}$, in agreement with the diagonal length of $128 \mathrm{~nm}$ expected for the folded tall rectangle. The width of the distribution further decreased until $50{ }^{\circ} \mathrm{C}$, where it remained unchanged and equal to $10 \mathrm{~nm}$. This trend is in agreement with the aforementioned qualitative observations. In contrast with the size distributions, the histograms of the modal height showed only two peaks suggesting that folding is a two-state process. However, this picture is incomplete because the modal height is very sensitive to the existence of holes inside a particle. Indeed, 
the two peaks should be considered as a signature of the existence of holes within a particle, $0.15 \mathrm{~nm}$ indicating some holes and $0.9 \mathrm{~nm}$ no holes. In any case, the modal height clearly indicated that in the range $59-55^{\circ} \mathrm{C}$, at least two distinct states coexisted. The histograms of the median height, less sensitive to the existence of holes, revealed at least four states along the folding pathway, at 0.3, 0.4, 0.5 and $0.8 \mathrm{~nm}$ (SI Figure S12).

From these data it is possible to select single particles that best represent the folding process at each temperature. To do so we fitted the modal and median height distributions to single and double gaussians to find their local maxima. We then looked for particles for which both heights were within $5 \%$ of the height corresponding to these maxima. Figure 3B displays, for each temperature, a set of most representative foldamers selected by this method. In the range $59-55^{\circ} \mathrm{C}$, two distinct states were associated to the two maxima observed in the height histograms. We have called these different sets of structures state I and II, and we note that state I at two different temperatures does not necessarily correspond to the same structure. This analysis confirmed the qualitative picture of folding provided above. The most likely foldamers at every temperature were: entangled coils $\left(70-65^{\circ} \mathrm{C}\right)$, half-width folded particles $\left(63{ }^{\circ} \mathrm{C}\right)$, full-width folded particles $\left(61{ }^{\circ} \mathrm{C}\right)$, a mixture of two types of full-width folded and broken rectangle particles $\left(59-57^{\circ} \mathrm{C}\right)$, a mixture of broken rectangles and fully folded particles $\left(55^{\circ} \mathrm{C}\right)$, and fully folded particles $\left(50-40^{\circ} \mathrm{C}\right)$. A complementary way of representing these data is to average, at each temperature, the particles after aligning them using an image registration algorithm (Figure 3C) against a prototypical rectangle particle at $40{ }^{\circ} \mathrm{C}$. This procedure is thus biased towards finding average rectangular shapes, as one notices when comparing averages at $69{ }^{\circ} \mathrm{C}$ and $61^{\circ} \mathrm{C}$ that look very similar, although whole-field AFM images appear significantly different to the eye (Figure 2). As a result, average images were more informative at lower temperatures where rectangular shapes were more apparent. In particular, they captured well the transition between $61{ }^{\circ} \mathrm{C}$, where full-width folded structures were majority, to $59{ }^{\circ} \mathrm{C}$, where broken rectangles became predominant. Moreover, average particles for states I and II at 57 and $55^{\circ} \mathrm{C}$, clearly showed 
the existence of two different folding states at these temperatures. In summary, the qualitative and quantitative analysis of AFM images are consistent and quantitative analysis provided statistically-significant measures of the structural intermediates that were essential to build up a coherent picture of the folding pathway.

\section{The melting and folding pathways differ at high temperature}

Third, we used our method to investigate the melting pathway of pre-folded DNA origamis. Figure 4 displays AFM images of single foldamers at different temperatures during heating. At first sight, two aspects are visually striking when comparing these images with those in Figure 2: the folded rectangles corresponding to the melting experiment do not display the weaving internal structure seen in Figure 2 and they look like squares, instead of rectangles (they are $80 \times 90 \mathrm{~nm}^{2}( \pm 5 \mathrm{~nm})$ instead of $\left.80 \times 100 \mathrm{~nm}^{2}\right)$. These two observations are consistent, the less the horizontally-running helices are bound to each other, the more the internal crossovers of the origami should become apparent, resulting in an enlargement of the dimensions along the vertical axes. We have observed a $10 \%$ variability in the size of the tall rectangles for experiments performed in different days and found $(74 \pm 7) \times(88 \pm 9) \mathrm{nm}^{2}$ (one standard deviation, SI Figure S5). In contrast, the variability is negligible for experiments performed the same day (SI Figure S6-8). Absolute particle sizes given in this work have thus to be taken with $10 \%$ precision and, as a consequence, the folded rectangles in Figure 2 and 4 are identical within experimental precision. Although the folding protocols in the folding and melting experiments were different (see Methods, below) we did not observe a correlation between the size and the folding protocol (SI Figure S5). We thus hypothesize that these changes in shape may come from some parameters that were not controlled in our experiment, especially humidity, and that may influence the adsorption of the sample onto the mica. We conclude that this variability did not influence, within experimental precision, the observed folding state at each temperature.

The data in Figure 4 show that the melting pathway was the reverse of the folding one 
until $61{ }^{\circ} \mathrm{C}$ : the central part of the rectangle melted at a lower temperature than the edges. In this range of temperatures, melting happened $2{ }^{\circ} \mathrm{C}$ above folding (SI Figure S11), a hysteresis compatible with melting curves of the bulk obtained using absorbance ${ }^{23}$ or fluorescence (SI Figure S13). However, this difference is within the $2{ }^{\circ} \mathrm{C}$ experimental precision of our method. In contrast, above $61^{\circ} \mathrm{C}$ the folding and melting pathways strongly differed: a new type of structure, called chain-like, was observed (Figure 4B). These chain-like structures reached $1 \mu \mathrm{m}$ across and were formed of links about $150 \mathrm{~nm}$ long. The number of links per particle clearly increased from 63 to $69^{\circ} \mathrm{C}$, in agreement with a progressive unwinding of dsDNA. At $70{ }^{\circ} \mathrm{C}$ we observed a mixture of these chain-like structures together with entangled coils, indicating that at very high temperature the chain-like structures were disrupted. This high-temperature hysteresis was not observed in melting curves of the bulk recored using a fluorescent DNA intercalator (SI Figure S13), indicating that the states observed at high temperature for folding and melting, although structurally very different, had a similar number of base pairs. Taken together, these results suggest that chain-like structures are out of equilibrium and correspond to single-stranded M13 loops connected by sets of staples forming dsDNA links with very low dissociation rates $\left(<0.025 \mathrm{~min}^{-1}\right.$ in the range $\left.65-69^{\circ} \mathrm{C}\right)$.

\section{The folding pathway can be controlled by the relative concentration of staples}

Fourth, we used the knowledge extracted from the previous experiments to try to control the folding pathway. As we have seen, the folding nucleation sites appeared to be thermodynamically controlled and corresponded to regions having higher melting temperatures, $T_{m}$. To test this hypothesis, and taking into account that $T_{m}$ depends on the concentration for a bimolecular reaction (see SI section 8), we folded the tall rectangle with different relative concentrations of the staples. Figure 5 shows AFM images of the folding process when the concentration of the central and border staples was changed from $52 \mathrm{nM}$ to, respectively, 670 and $1.2 \mathrm{nM}$. In these conditions the folding pathway was dramatically changed and 
proceeded this time from the center towards the edges. Centre-folded structures bearing a $72 \times 45 \mathrm{~nm}^{2}$ double-stranded rectangular region at the center of the structure were observed at $59{ }^{\circ} \mathrm{C}$. This region increased its size to $72 \times 85 \mathrm{~nm}^{2}$ to form an advanced centre-folded structure at $55{ }^{\circ} \mathrm{C}$. At $40{ }^{\circ} \mathrm{C}$ normal rectangles were observed on top of a rugged surface, probably due to staple adsorption. FInally, the onset of folding was shifted from 63 to $59{ }^{\circ} \mathrm{C}$, a trend that was also observed in bulk (SI Figure S14). These results confirm that the folding pathway is controlled thermodynamically and that the nucleation sites correspond to the staples with the highest melting temperature. They moreover open the way to the design of nanostructures that could be controlled by the folding pathway.

To understand the effect of staple concentration on the stability of the nucleation site let us consider the reaction of one staple, $\mathrm{S}$, with the scaffold, T. We suppose that the staple has two identical biding sites and call $\mathrm{C}$ the complex of $\mathrm{T}$ bound to one binding site of the staple, D the complex bound to two staples $\mathrm{S}$, and $\mathrm{O}$ the well-folded nucleation site where one staple binds at its two sites. In the absence of cooperativity we can write

$$
\begin{aligned}
\mathrm{S}+\mathrm{T} & \stackrel{K_{1}}{\rightleftharpoons} \mathrm{C} \\
\mathrm{C}+\mathrm{S} & \stackrel{K_{1}}{\rightleftharpoons} \mathrm{D} \\
\mathrm{C} & \stackrel{K_{2}}{\rightleftharpoons} \mathrm{O},
\end{aligned}
$$

where $K_{i}$ is the corresponding equilibrium constant. When the total concentration of staples $S_{0}$ is low, we can neglect reaction 2 and the equilibrium concentration of nucleation site, $O$, scales as $O \sim K_{1} S_{0}$, which is related to $T_{m}$. When the staple concentration is in excess, $O$ increases with $S_{0}$ only if $K_{1} S_{0}<1$. For $K_{1} S_{0}>1$ reaction 2 competes with reaction 3 (SI Section 8). In other words, decreasing the concentration of the edge staples to $1.2 \mathrm{nM}$ decreases the stability of edge nucleation sites and increasing the concentration of central staples to $670 \mathrm{nM}$ increases the stability of central nucleation sites as far as $K_{1} S_{0}<1$. 
Our observations suggest that both conditions applied: the increase of the central staples increased the folding temperature of the central region from 55 to $59{ }^{\circ} \mathrm{C}$ while the decrease of the edge staples decreased folding temperature of the edges from 61 to $55^{\circ} \mathrm{C}$.

\section{Single-molecule and bulk folding experiments agree}

Finally, we checked that the folding pathway drawn from single-molecule data was in agreement with data acquired using standard bulk techniques. To do so, following reference 17, the extent of the origami folding reaction was followed using a fluorescent DNA binder. In these bulk experiments, to increase the signal, the scaffold concentration was 10-fold (10 nM) that of AFM experiments, while the staple concentration remained unchanged (52 nM). For the bimolecular reaction of a scaffold with a staple to form a dsDNA, one expects the extent of the reaction to be controlled by the highest concentration (here the staples). As a result, the 10-fold increase in scaffold concentration should induce negligible changes. Figure 6 shows folding curves corresponding to the median height from AFM data and to fluorescence for bulk experiments (see also SI Figure S10). Their good agreement is remarkable, indicating that our method, despite relying on an adsorption step, does provide representative snapshots of the folding pathway in bulk. The folding temperatures are 59 and $61.5^{\circ} \mathrm{C}$, for AFM and bulk respectively. The gap between the two techniques is lower at high and medium folding, $1-2{ }^{\circ} \mathrm{C}$, than at low folding, $4-7^{\circ} \mathrm{C}$. There are three reasons that may explain this difference. First, the presence of the fluorescent binder is reported to stabilize dsDNA structures by $1-2{ }^{\circ} \mathrm{C} .{ }^{24,25}$ Second, the bulk technique is proportional to the concentration of dsDNA, while AFM is sensitive to the formation of structures large enough to be detected (typically $10 \mathrm{~nm}$ ). Moreover, the order parameter chosen here, the median height, particularly suffers this bias, as visual inspection of AFM folding data at $65{ }^{\circ} \mathrm{C}$ (Figure 2) indicates that some folding has already occurred while the median histogram (Figure 3) does not show any changes. This feature suggests that the bulk experiment will better detect structural changes at low folding while the AFM one will do so for higher folding values, 
in agreement with the data. Finally, during sample preparation some uncontrolled cooling could occur, specially at high temperatures, although our protocol was designed to minimize these problems (Figure 1 and SI Section 10). In contrast with our data, when the tall rectangle was adsorbed on mica and its mechanism studied in situ by $\mathrm{AFM}^{15}$ folding could not be obtained and melting yielded a significantly higher melting temperature. As expected, the interaction between the origami and the mica surface in those experiments over-stabilized the folded state, specially at low folding.

\section{Conclusion}

In summary, we have developed a method to analyze a large population of origamis, during their folding, that combines, for the first time, statistical analysis on the ensemble and single molecule resolution. It relies on freezing the conformation of hundreds of individual origamis by adsorption on a mica surface at each temperature of the folding or melting protocol. We have proposed an automated image analysis procedure allowing to extract, for each temperature, the statistical distribution of morphological traits (length, height), an average morphology as well as the most representative one. We think that such a method will be useful not only for or a better understanding of DNA origami folding and other highly complex self-assembly processes (e.g., DNA-mediated particle assembly) but also to quantitatively characterize and compare the performance of emerging methods in DNA nanofabrication, where a simple eye inspection of arbitrarily chosen structures at equilibrium is not enough. For instance, in this work we have used this method to analyze the folding pathway of rectangular DNA origamis with single-molecule resolution. For this particular morphology we have identified well-defined intermediates corresponding to equilibrium structures and shown that, under standard conditions, folding occurred from the edges to the center, while melting processed through a reverse pathway. In a practical perspective, this method can be used to determine temperatures leading to reproducible structures at equilibrium with 
peculiar morphologies that are different from the fully folded one at room temperature. We have shown that it can also be exploited to optimize the folding protocol by focusing the cooling procedure where folding actually takes place, resulting in a gain in robustness (fewer intermediate steps) and time (the folding protocol can be significantly shortened). We thus anticipate this method to be useful not only to optimize fabrication procedures but also for troubleshooting analysis of misfolded origamis. However, the interest of our method is not limited to highly-resolved inspection of origami morphologies as a function of temperature. It has allowed us to extract general and new information on the folding pathway of DNA origamis. First, we have demonstrated that folding happens under thermodynamic control, making DNA origami a particularly reliable tool for building reproducible structures by bottom-up assembly. Second, we have shown that the pathway is primarily defined by the distribution of the melting temperature of staples bound to the scaffold, the origamis folding from high to low melting temperature area. One way to modify the melting temperature distribution consists in changing the sequence of the staples but this implies a complex adaptation of the scaffold/staple sequence composition for each given structure. In this work, we have demonstrated an experimentally straightforward alternative to affect the melting temperature profile along the folded origami structure without modifying the sequence of neither the scaffold nor the staples. By simply changing the concentration ratio of staples, we have shown that we can increase or decrease the melting temperature of a set of target staples, resulting in a direct control of the origami folding pathway. This constitutes an interesting method to rationally design DNA nanostructures that can be actuated by temperature, with well-defined dynamic morphological responses at predefined positions. Overall, by bringing both a powerful tool of DNA nanostructure analysis and new information of the DNA origami folding pathway, we think this work will contribute to the rationalization of current and future methods to generate highly organized, morphologically sophisticated and dynamically addressable nanostructures generated according to DNA base pairing rules. 


\section{Methods}

\section{Sample preparation}

All AFM experiments were performed with a solution of $1 \mathrm{nM}$ of M13mp18 (NEB) in TAE 1X and $12.5 \mathrm{mM}$ magnesium acetate buffer together with the 'Tall Rectangle' staples, the sequences of which can be found in Figure S38 of the SI of ref. 3. For standard conditions we used $52 \mathrm{nM}$ of each of the staples. For Figure 5 we used a mixture of $1.2 \mathrm{nM}$ of the outermost row staples (4 on the northern edge and 4 on the southern edge, the sequences are provided in the SI) and $670 \mathrm{nM}$ of the 9 rows of central staples. In all cases the west and east edge staples were not added to minimize the stacking interaction between folded structures on the mica surface. $150 \mu \mathrm{L}$ aliquots of this origami solution were distributed in $500 \mu \mathrm{L}$ tubes. The same number of aliquots of pure buffer were also prepared.

\section{Temperature ramping protocol}

Origami and buffer solutions were subjected to a thermal ramp in a Peqstar 2x thermocycler (Peqlab). For the folding experiment the ramp consisted of an initial 10 min-long step at $95{ }^{\circ} \mathrm{C}$ followed by a decrease of $1{ }^{\circ} \mathrm{C}$ every 15 minutes from $70{ }^{\circ} \mathrm{C}$ to $40{ }^{\circ} \mathrm{C}$. For the melting experiment we first prepared the fully formed origami with a standard protocol (10 min at $70{ }^{\circ} \mathrm{C}$, followed by a decrease of $1{ }^{\circ} \mathrm{C}$ every 2 min from $70{ }^{\circ} \mathrm{C}$ to $40{ }^{\circ} \mathrm{C}$ ) followed by a 10 min-long step at $40{ }^{\circ} \mathrm{C}$, a fast increase of $1{ }^{\circ} \mathrm{C}$ every 2 min from $40{ }^{\circ} \mathrm{C}$ to $55^{\circ} \mathrm{C}$ and then a slower increase of $10 \mathrm{~min} /{ }^{\circ} \mathrm{C}$ until $70{ }^{\circ} \mathrm{C}$. In both cases, for a given temperature, a c.a. $3 \times 5 \mathrm{~mm}^{2}$ piece of mica cut with scissors was freshly cleaved and immersed in the origami solution at the end of the step for $1 \mathrm{~min}$. Immersion of a cold piece of mica inside a hot solution is expected to induce a negligible temperature change (SI Section 10). The mica was then quickly taken out and dipped into a buffer solution having the same temperature for $30 \mathrm{~s}$. The mica was subsequently removed from the buffer, delicately washed with $1 \mathrm{~mL}$ of Milli-Q water at room temperature (SI Figure S9), quickly dried under a compressed air 
stream and stuck on a metal disc using double-sided tape prior to AFM observation.

\section{AFM imaging}

AFM images were acquired using an Agilent Technologies 5100 Scanning Probe Microscope in tapping mode in air. We used AppNano FORT silicon probes with $43-81 \mathrm{kHz}$ nominal frequency and 0.6-3.7 N/m force constant. The amplitude set point was typically $15-20 \mathrm{~V}$ and the scan rate 1.5 lines/s. Raw AFM images were baseline corrected using Gwyddion: we sequentially performed a "Flatten base" and a "Line-wise sliding median correction" until the corrected image did not change further (in our hands three "Flatten base" and two "Linewise sliding"). The "Flatten base" subtracts a global polynomial fit to the image excluding aberrant data points. The "Line-wise sliding" calculates the median of the pixel intensity of a line and its adjacent line and shifts the pixels such that the medians are equal. This process is carried on the entire image. Note that the relative heights within a sample preparation are consistent but absolute values (for example de height of dsDNA) from sample to sample may vary by $\pm 0.2 \mathrm{~nm}$. Because our AFM images were obtained on dried samples the height of dsDNA was $1.2 \mathrm{~nm}$ instead of $2 \mathrm{~nm}$.

\section{Image segmentation}

32 bit corrected AFM images were segmented using a semi-automatic custom made procedure in ImageJ 2.0. First, images were binarized using an optimal threshold determined manually. To do so, every image was segmented with a systematic range of thresholds in $0.05 \mathrm{~nm}$ steps, the outline of the detected particles was overlaid over the AFM image and the optimal threshold was determined visually. This threshold depended on the height of the particles in the sample and thus on the temperature of the folding experiment. The optimal thresholds (in $\mathrm{nm}$ ) for the folding experiment were: 0.5 for $T \leq 50,0.3$ for $55 \leq T \leq 59$, 0.2 for

$T=61$ and 0.1 for $T \geq 63$, where $T$ is the temperature in ${ }^{\circ} \mathrm{C}$. The thresholds for the melting experiment were identical except for $T \geq 63$ where the threshold was $0.15 \mathrm{~nm}$. Binary images 
were eroded once and dilated twice, except for $T \leq 50$ were erosion was done twice followed by 3 dilations. Subsequently, for every image, particles were detected using the function "Analyze Particles". Particles smaller than $4.7 \times 10^{3} \mathrm{~nm}^{2}$ were discarded for all samples and particles larger than $1.6 \times 10^{4} \mathrm{~nm}^{2}$ were discarded for $T \leq 55$, i.e. where origami were almost fully formed, to remove particles resulting from two rectangles touching each other. For comparison, the area of a tall rectangle was $8 \times 10^{3} \mathrm{~nm}^{2}$. Particles touching the borders of the image were discarded and no constrain on the circularity of the particles was used. This procedure is available in SI section 11.

\section{Fluorescence melting curves}

Melting curves of rectangular origami in bulk were obtained through a method adapted from ref. 17. The scaffold:staples concentration was 10:52 nM. Experiments were performed in the same buffer as AFM experiments supplemented with $160 \mathrm{nM}$ SybrGreen I, i.e. there is less than 1 SybrGreen molecule per $400 \mathrm{bp}$. The sample was heated to $90{ }^{\circ} \mathrm{C}$ and cooled at a rate of $1{ }^{\circ} \mathrm{C} / 10 \mathrm{~min}$ or $1{ }^{\circ} \mathrm{C} / 30 \mathrm{~min}$. Control experiments containing the scaffold and the staples alone were also performed. The order parameter was obtained by subtracting staples fluorescence from origami fluorescence and normalizing.

\section{Acknowledgement}

This work was funded by European comission FET-STREP under award Ribonets and by ANR jeunes chercheurs program under award Dynano. We thank X. Tassart for preliminary

experiments and J.C. Galas, A. Zadorin, A. Genot, Y. Rondelez and J.-P. Aimé for insightful discussions. 


\section{Supporting Information Available}

Additional figures S1-S14, table S1, a discussion of the effect of staple concentration on melting temperature, an evaluation of the thermal effect, the Matlab and ImageJ routines for image segmentation and the sequences of staples used.

This material is available free of charge via the Internet at http://pubs.acs.org/.

\section{References}

(1) Seeman, N. C. Nucleic-acid Junctions and Lattices. J. Theor. Biol. 1982, 99, 237-247.

(2) Chen, J.; Seeman, N. C. Synthesis from DNA of a Molecule with the Connectivity of a Cube. Nature 1991, 350, 631-633.

(3) Rothemund, P. W. K. Folding DNA to Create Nanoscale Shapes and Patterns. Nature 2006, 440, 297-302.

(4) Han, D.; Pal, S.; Nangreave, J.; Deng, Z.; Liu, Y.; Yan, H. DNA Origami with Complex Curvatures in Three-Dimensional Space. Science 2011, 332, 342-346.

(5) Pinheiro, A. V.; Han, D.; Shih, W. M.; Yan, H. Challenges and Opportunities for Structural DNA Nanotechnology. Nature Nanotech. 2011, 6, 763-772.

(6) Douglas, S. M.; Dietz, H.; Liedl, T.; Hogberg, B.; Graf, F.; Shih, W. M. Self-assembly of DNA into Nanoscale Three-dimensional Shapes. Nature 2009, 459, 414-418.

(7) Dietz, H.; Douglas, S. M.; Shih, W. M. Folding DNA into Twisted and Curved Nanoscale Shapes. Science 2009, 325, 725.

(8) Andersen, E. S.; Dong, M.; Nielsen, M. M.; Jahn, K.; Subramani, R.; Mamdouh, W.; Golas, M. M.; Sander, B.; Stark, H.; Oliveira, C. L. P.; Pedersen, J. S.; Birkedal, V.; Besenbacher, F.; Gothelf, K. V.; Kjems, J. Self-assembly of a Nanoscale DNA Box with a Controllable Lid. Nature 2009, 459, 73-76. 
(9) Wei, X.; Nangreave, J.; Jiang, S.; Yan, H.; Liu, Y. Mapping the Thermal Behavior of DNA Origami Nanostructures. J. Am. Chem. Soc. 2013, 135, 6165-6176.

(10) Benson, E.; Mohammed, A.; Gardell, J.; Masich, S.; Czeizler, E.; Orponen, P.; Hogberg, B. DNA Rendering of Polyhedral Meshes at the Nanoscale. Nature 2015, 523, 441-4.

(11) Endo, M.; Yang, Y.; Sugiyama, H. DNA Origami Technology for Biomaterials Applications. Biomaterials Science 2013, 1, 347-360.

(12) Pfitzner, E.; Wachauf, C.; Kilchherr, F.; Pelz, B.; Shih, W. M.; Rief, M.; Dietz, H. Rigid DNA Beams for High-Resolution Single-Molecule Mechanics. Angew. Chem. Intl. Ed. 2013, 52, 7766-7771.

(13) Langecker, M.; Arnaut, V.; Martin, T. G.; List, J.; Renner, S.; Mayer, M.; Dietz, H.; Simmel, F. C. Synthetic Lipid Membrane Channels Formed by Designed DNA Nanostructures. Science 2012, 338, 932-936.

(14) Kuzyk, A.; Schreiber, R.; Fan, Z.; Pardatscher, G.; Roller, E.-M.; Hogele, A.; Simmel, F. C.; Govorov, A. O.; Liedl, T. DNA-based Self-assembly of Chiral Plasmonic Nanostructures with Tailored Optical Response. Nature 2012, 483, 311-314.

(15) Song, J.; Arbona, J.-M.; Zhang, Z.; Liu, L.; Xie, E.; Elezgaray, J.; Aime, J.-P.; Gothelf, K. V.; Besenbacher, F.; Dong, M. Direct Visualization of Transient Thermal Response of a DNA Origami. J. Am. Chem. Soc. 2012, 134, 9844-9847.

(16) Pastré, D.; Piétrement, O.; Fusil, S.; Landousy, F.; Jeusset, J.; David, M.-O.; Hamon, L.; Le Cam, E.; Zozime, A. Adsorption of DNA to Mica Mediated by Divalent Counterions: A Theoretical and Experimental Study. Biophys. J. 2003, 85, 2507-2518.

(17) Sobczak, J.-P. J.; Martin, T. G.; Gerling, T.; Dietz, H. Rapid Folding of DNA into Nanoscale Shapes at Constant Temperature. Science 2012, 338, 1458-1461. 
(18) Wei, X.; Nangreave, J.; Liu, Y. Uncovering the Self-Assembly of DNA Nanostructures by Thermodynamics and Kinetics. Acc. Chem. Res. 2014, 47, 1861-1870.

(19) Arbona, J. M.; Elezgaray, J.; Aim, J. P. Modelling the Folding of DNA Origami. Europhys. Lett. 2012, 100, 28006.

(20) Dunn, K. E.; Dannenberg, F.; Ouldridge, T. E.; Kwiatkowska, M.; Turberfield, A. J.; Bath, J. Guiding the Folding Pathway of DNA Origami. Nature 2015, 525, 82-86.

(21) Bae, W.; Kim, K.; Min, D.; Ryu, J. K.; Hyeon, C.; Yoon, T. Y. Programmed Folding of DNA Origami Structures Through Single-molecule Force Control. Nat Commun 2014, 5,5654 .

(22) Allemand, J. F.; Bensimon, D.; Jullien, L.; Bensimon, A.; Croquette, V. pH-dependent Specific Binding and Combing of DNA. Biophys. J. 1997, 73, 2064-2070.

(23) Arbona, J. M.; Aime, J. P.; Elezgaray, J. Cooperativity in the Annealing of DNA Origamis. J Chem Phys 2013, 138, 015105.

(24) Monis, P. T.; Giglio, S.; Saint, C. P. Comparison of SYTO9 and SYBR Green I for Realtime Polymerase Chain Reaction and Investigation of the Effect of Dye Concentration on Amplification and DNA Melting Curve Analysis. Anal. Biochem. 2005, 340, 24-34.

(25) Gudnason, H.; Dufva, M.; Bang, D. D.; Wolff, A. Comparison of Multiple DNA Dyes for Real-time PCR: Effects of Dye Concentration and Sequence Composition on DNA Amplification and Melting Temperature. Nuc. Acids Res. 2007, 35, e127. 

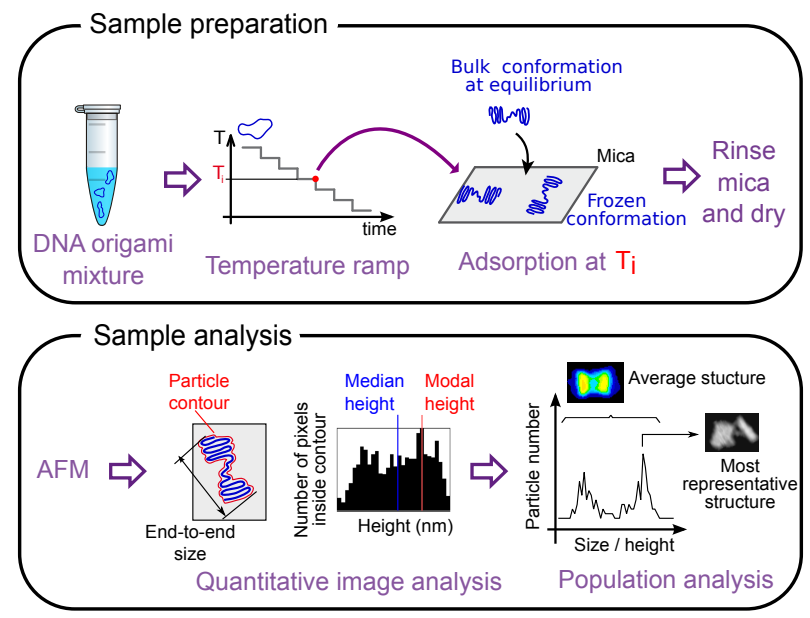

Figure 1: Sketch of the method used to characterize equilibrium origami structures during folding at the single molecule level. Replicate origami solutions (scaffold and staples) were submitted to a slow temperature ramp. At a desired temperature $\mathrm{T}_{i}$, a piece of mica was immersed in the solution to freeze the equilibrium conformations on its surface by adsorption. Rinsing of adsorbed samples on mica was performed at $T_{i}$. After drying, AFM images were acquired. Image analysis yielded particle contours and quantitative data, such as the endto-end size, and the modal and median height, for every foldamer. Distributions of these parameters were obtained at each temperature, allowing to extract the average and the most representative structure. 

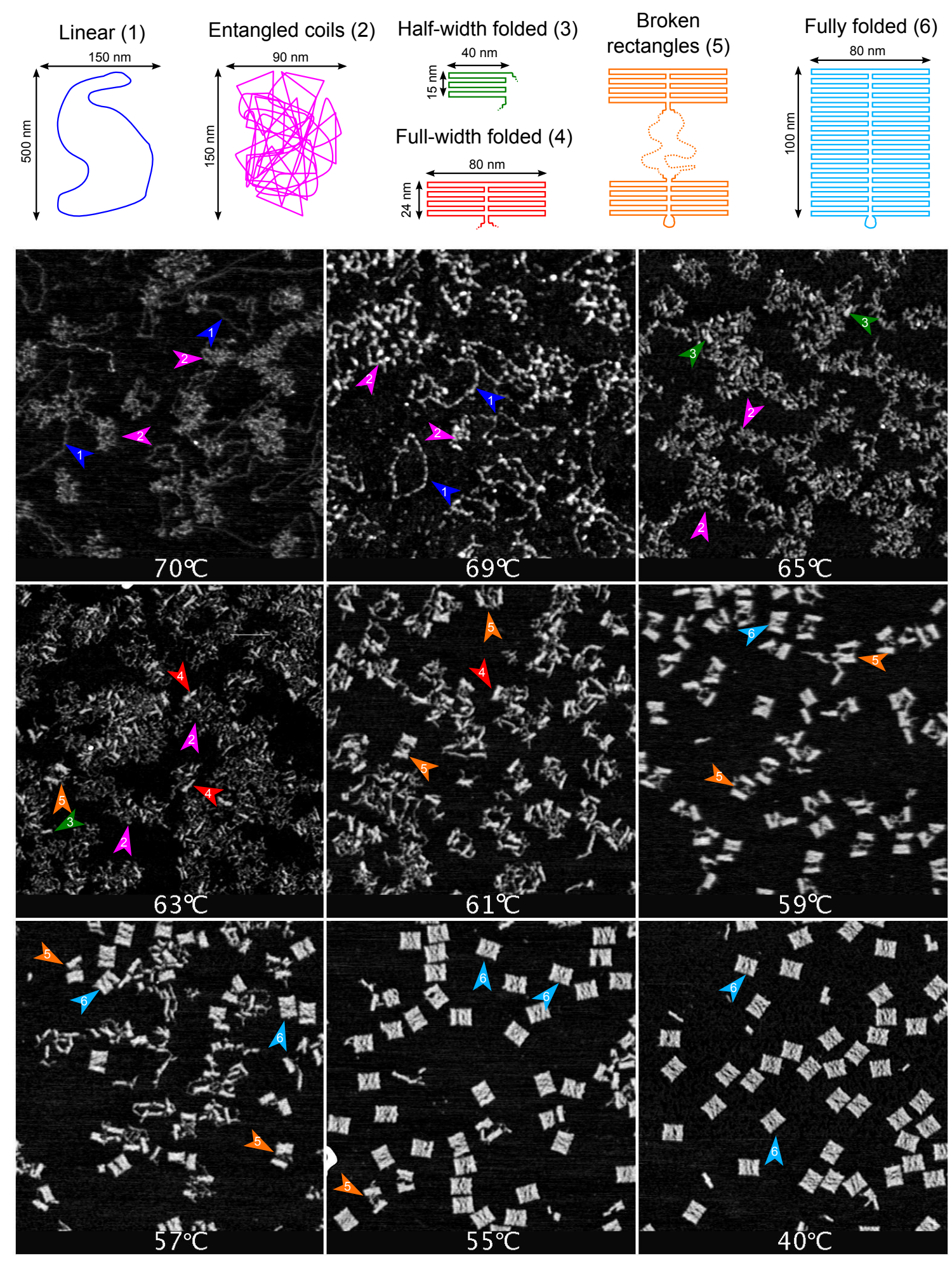

Figure 2: DNA origami structures folding during a cooling ramp of $1{ }^{\circ} \mathrm{C} / 15 \mathrm{~min}$. Scaffold $1 \mathrm{nM}$, staples $52 \mathrm{nM}$. Top: sketches of the structural types observed. Bottom: AFM snapshots at different temperatures, each image corresponds to a different sample following the same folding protocol. Colored and numbered arrows indicate structures belonging to the structural types of identical color and number sketched in the top panel. All images are $1.5 \times 1.5 \mu \mathrm{m}^{2}$ with a height range of $0-1.3 \mathrm{~nm}$. 


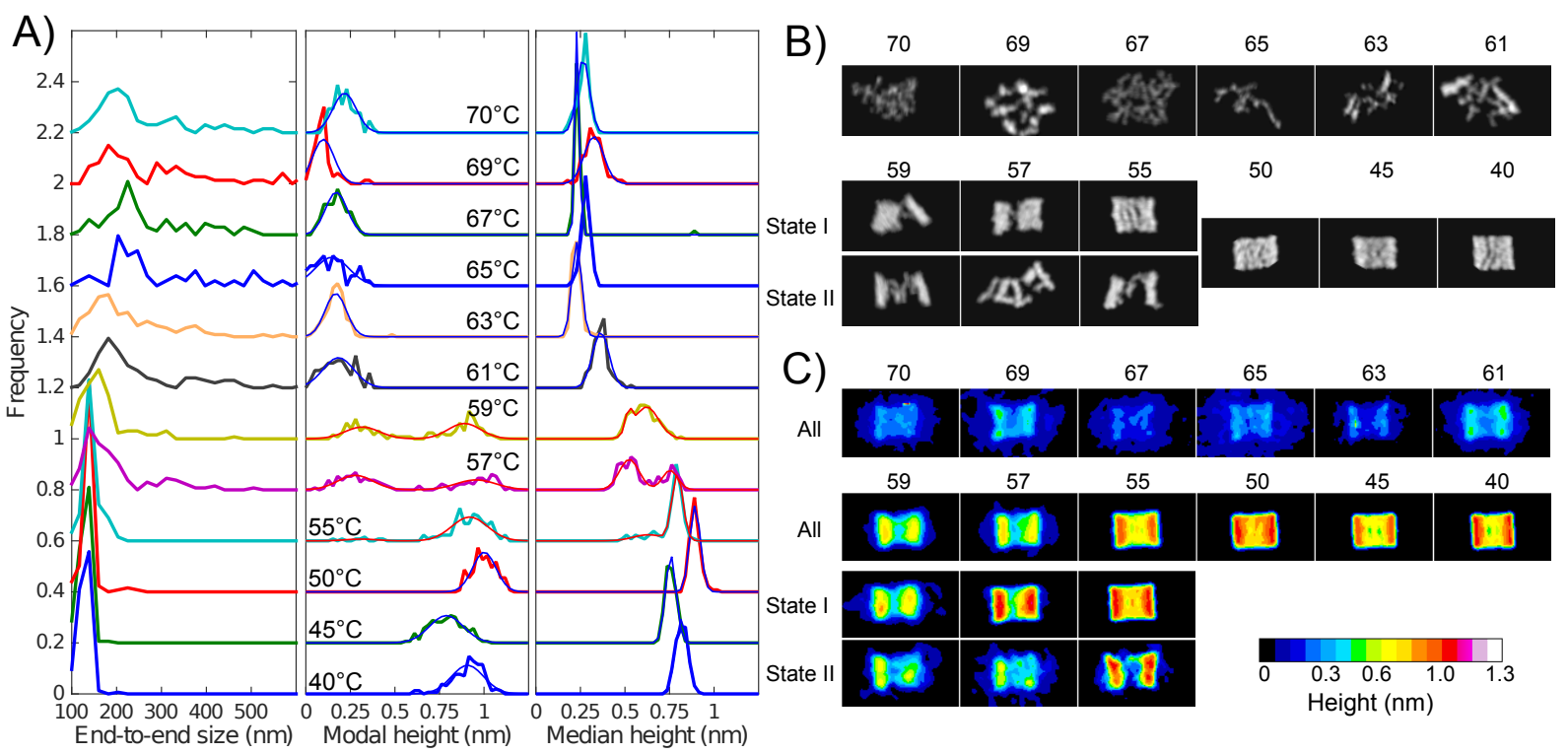

Figure 3: Population analysis of an ensemble of DNA origami particles folding while cooling into a tall rectangle. (A) Histograms of the end-to-end length, the modal height, and the median height of particles extracted from the whole field of view $\left(3 \times 3 \mu \mathrm{m}^{2}\right)$ of the images in Figure 2 (See SI Figure S1, S2) at different temperatures, plots are offseted for clarity. Thin blue and red lines correspond to single and double gaussian fits, respectively. Between 50 and 150 particles were analyzed for each temperature. (B) AFM images of representative particles close to the maxima of the median height distribution and (C) average AFM images at each temperature. State I and II correspond to the two populations found in the range $55-59{ }^{\circ} \mathrm{C}$. Images are $146 \times 88 \mathrm{~nm}^{2}$. Cooling rate $1^{\circ} \mathrm{C} / 15 \mathrm{~min}$. 


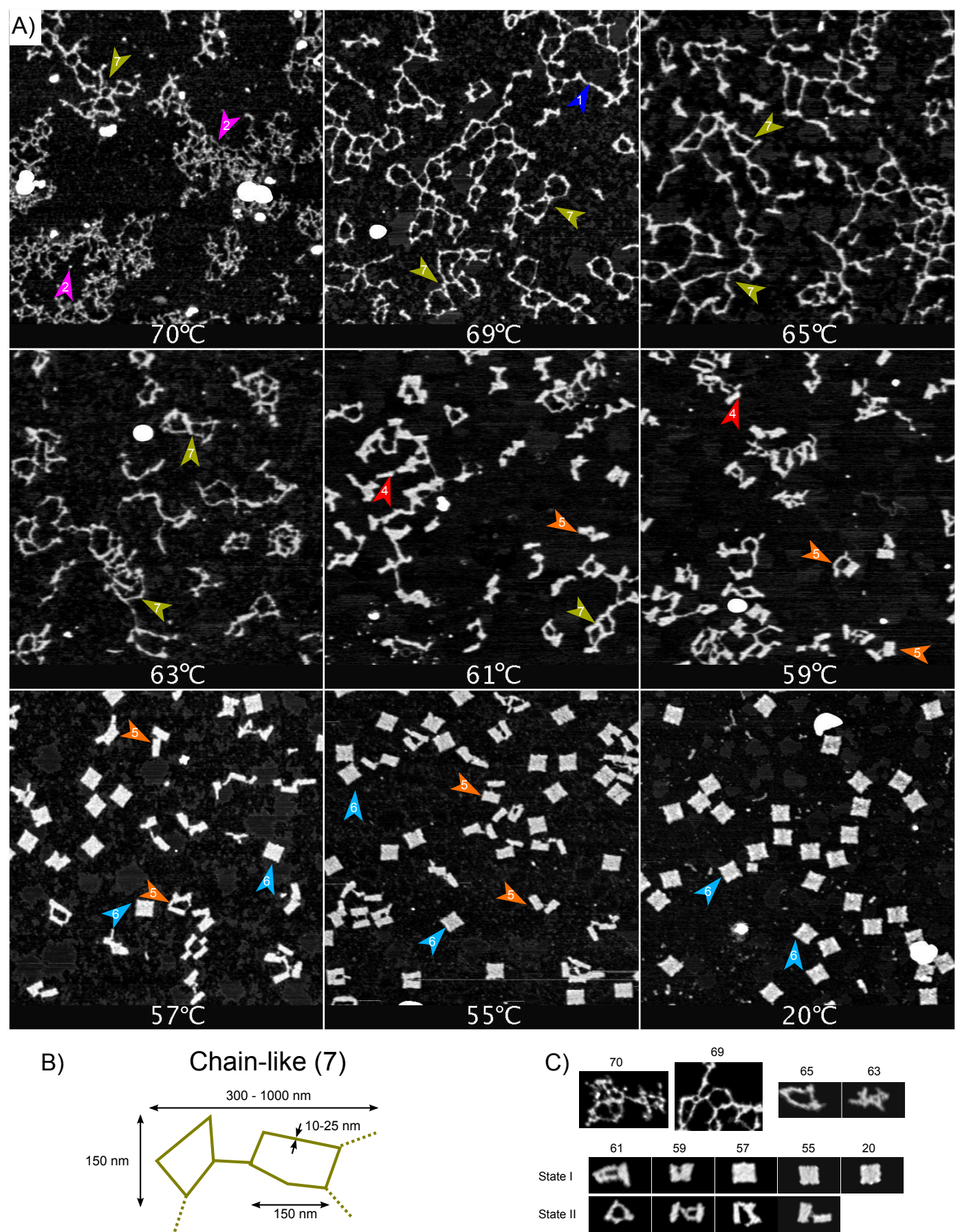

Figure 4: DNA origami structures melting during a heating ramp of $1{ }^{\circ} \mathrm{C} / 10$ min. A) AFM snapshots at different temperatures, each image corresponds to a different sample following the same melting protocol. The images are displayed from high to low temperature to facilitate comparison with Figure 2. Colored and numbered arrows indicate structures belonging to the structural types of identical color and number sketched in Figure 2 and panel B. All images are $1.5 \times 1.5 \mu \mathrm{m}^{2}$ with a height range of $0-1.3 \mathrm{~nm}$. B) sketch of a new structural type observed during heating. C) AFM images of representative particles close to the maxima of the median height distribution. Images are $146 \times 88 \mathrm{~nm}^{2}$ except 70 and 69 that are larger, but drawn at the same scale. 

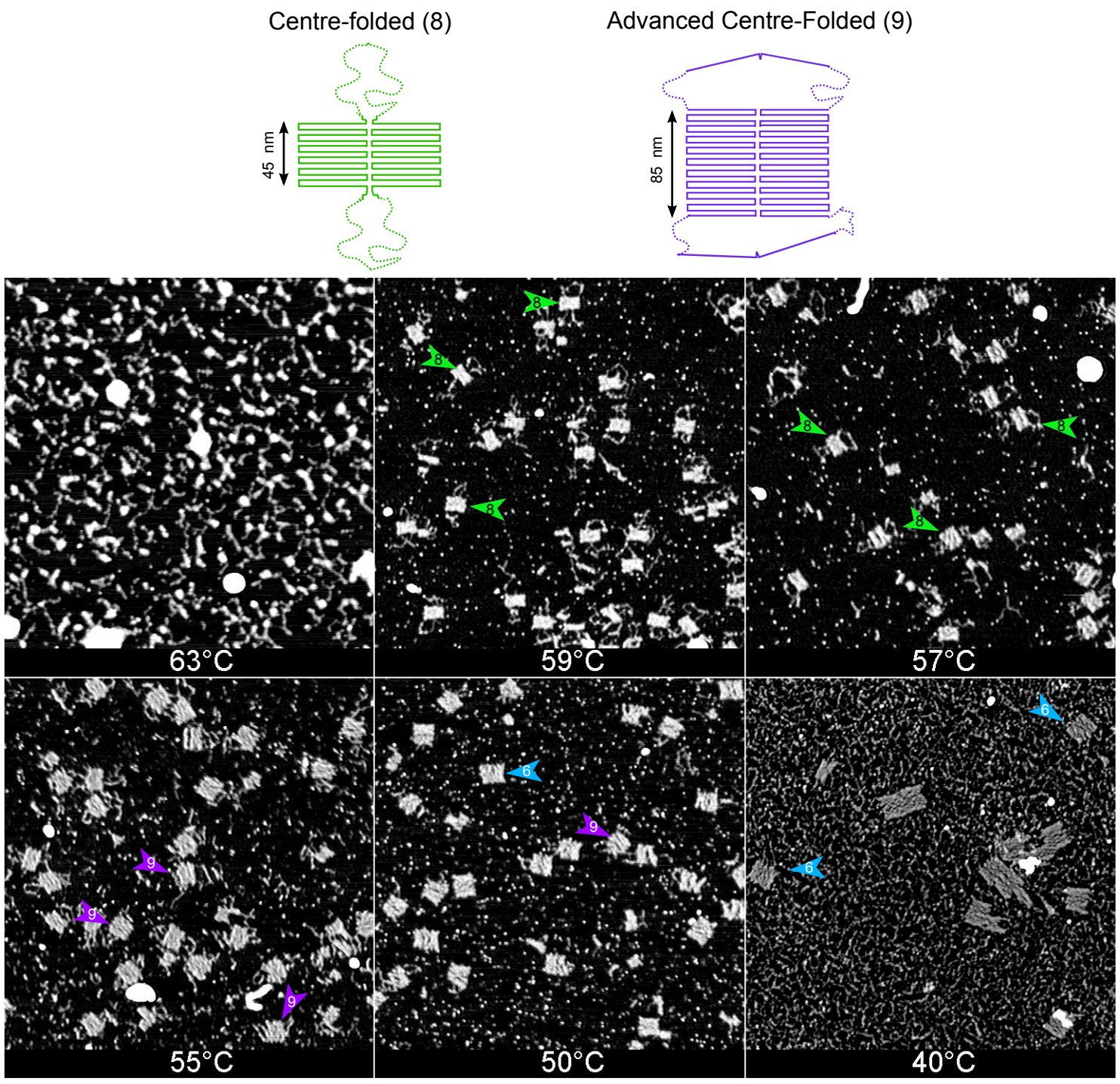

Figure 5: The folding pathway can be controlled by changing the relative concentration of staples. DNA origami structures folding during a cooling ramp of $1{ }^{\circ} \mathrm{C} / 30 \mathrm{~min}$. Scaffold $1 \mathrm{nM}$, edge staples $1.2 \mathrm{nM}$, central staples $670 \mathrm{nM}$. Top: sketches of the new structural types observed. Bottom: AFM snapshots at different temperatures. Colored and numbered arrows indicate structures belonging to the structural types of identical color and number sketched in the top panel and in Figure 2. All images are $1.5 \times 1.5 \mu \mathrm{m}^{2}$ with a height range of $0-1.3 \mathrm{~nm}$. 


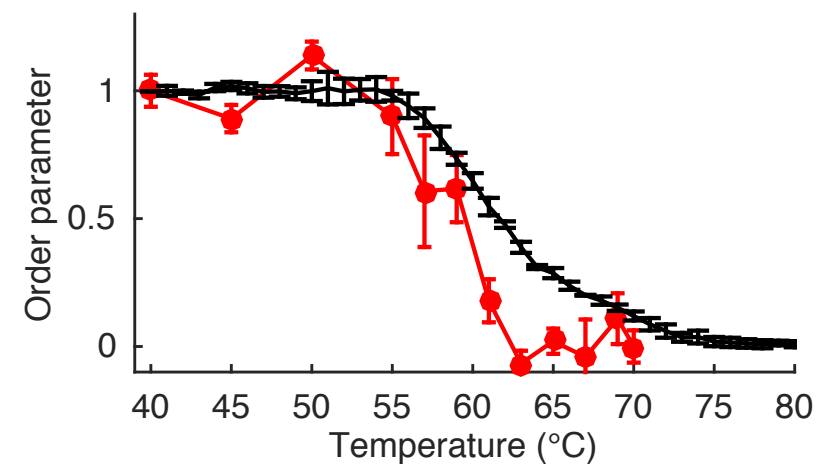

Figure 6: Single-molecule and bulk measurements are in good agreement. Folding curves with the AFM method described here (red disks) and with a standard macroscopic method relying on the fluorescence of a DNA binder ${ }^{17}$ (black line). The order parameter for AFM data is the average of the median height for either folding. The order parameter for macroscopic data is the fluorescence of an origami solution minus that of a staples solution during melting. Staples $52 \mathrm{nM}$, scaffold $1 \mathrm{nM}$ for AFM and $10 \mathrm{nM}$ for bulk.

\section{Graphical TOC entry}

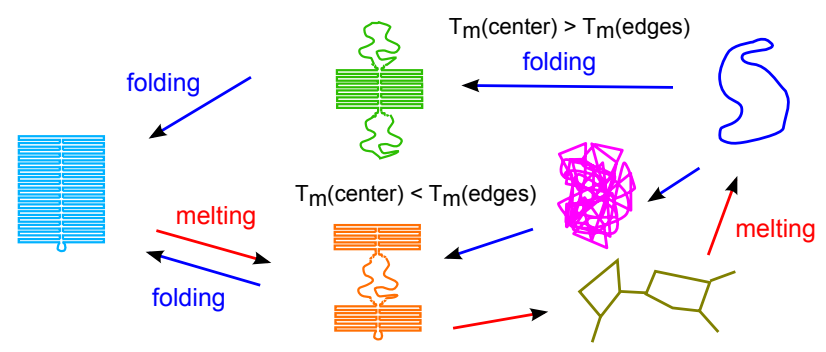




\title{
Supplementary information for Observing and Controlling the Folding Pathway of DNA Origami at the Nanoscale
}

\author{
Jonathan Lee Tin $\mathrm{Wah}^{a, b}$, Christophe David ${ }^{c}$, Sergii Rudiuk ${ }^{d, e, f}$, \\ Damien Baigl ${ }^{d, e, f}$ and André Estevez-Torres ${ }^{a, b}$
}

${ }^{a}$ Université Pierre et Marie Curie, Laboratoire Jean Perrin, 4 place Jussieu, 75005 Paris, France. ${ }^{b}$ CNRS, UMR 8237. ${ }^{c}$ Laboratoire de photonique et de nanostructures, CNRS, route de Nozay, 91460 Marcoussis, France ${ }^{d}$ Ecole Normale Supérieure -PSL Research University, Department of Chemistry, 24 Rue Lhomond, 75005, Paris, France. ${ }^{e}$ Sorbonne Universités, UPMC Univ Paris 06 PASTEUR, 75005, Paris, France. ${ }^{f}$ CNRS, UMR 8640 PASTEUR, 75005, Paris, France

\section{Contents}

1 Detected particles during folding 3

2 Detected particles during melting $\quad 5$

3 Variability of tall rectangle size as measured by AFM in different days 7

4 Reproducibility of AFM images during folding $\quad 8$

5 Effect of excess water rinsing on folded rectangle integrity 11

6 Skipping a range of temperatures during folding $\quad 12$

7 Quantitative analysis of AFM images 13

7.1 Quantitative analysis of melting . . . . . . . . . . . . . . . 13

7.2 Comparison of folding and melting . . . . . . . . . . . . . . 14

8 Dependence of the melting temperature on the staple concentration $\mathbf{1 5}$

8.1 Effect of staple concentration on nucleation site concentration . . . . . . 15

8.2 Estimating the change in $T_{m} \ldots \ldots \ldots \ldots$

9 Melting curves in bulk $\quad 18$

9.1 Influence of the temperature rate . . . . . . . . . . . . . . . 18

9.2 Influence of relative staple concentration . . . . . . . . . . . . . . 18

10 Evaluation of the thermal effect of dipping mica on a solution of different temperature 
11 Image segmentation procedure 


\section{Detected particles during folding}

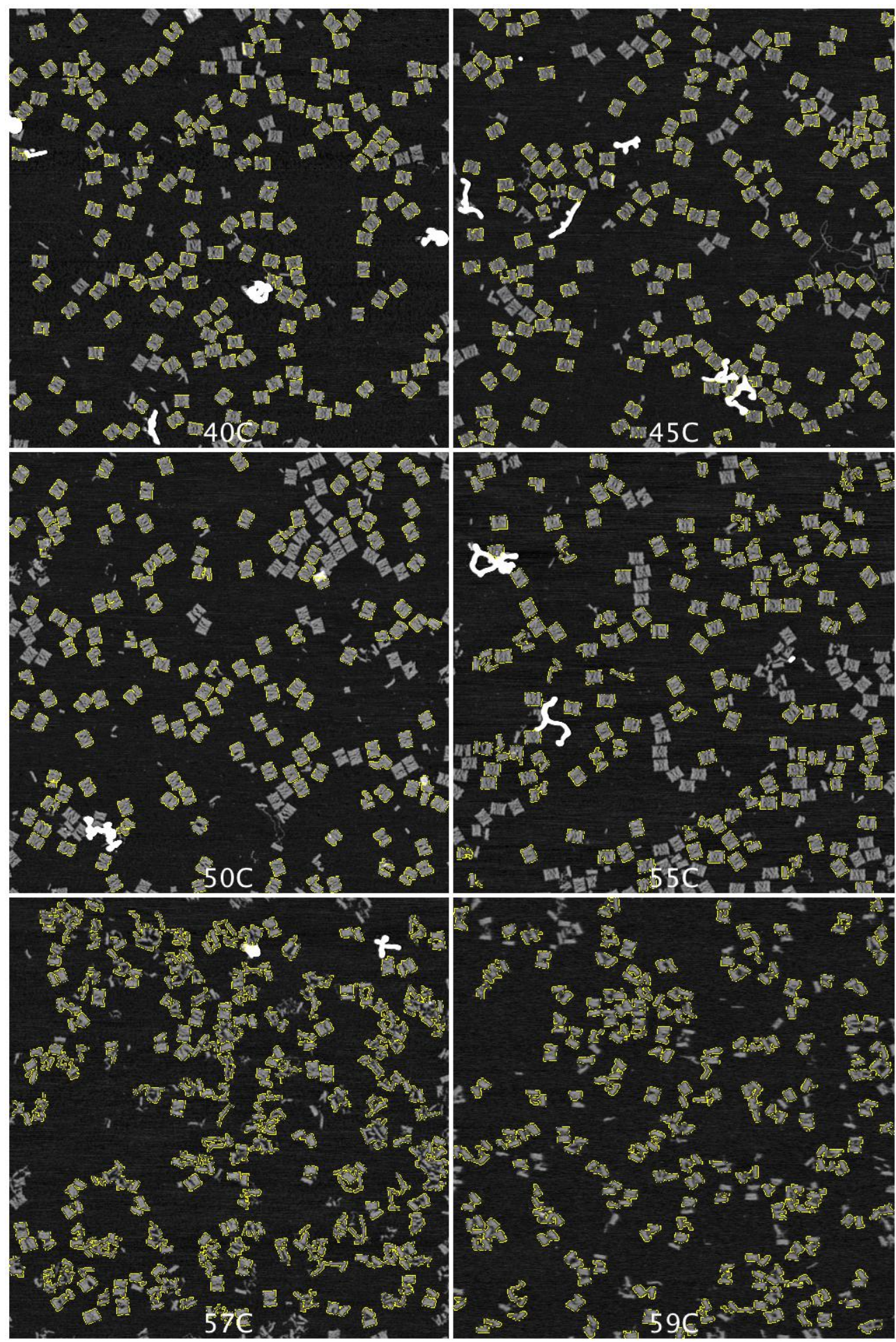

Figure S1: Detected particles (perimeter in yellow) during DNA tall rectangle folding while cooling. Data associated to Figure 2 and 3 in the Main Text. All images are $3 \times 3 \mu \mathrm{m}^{2}$. 


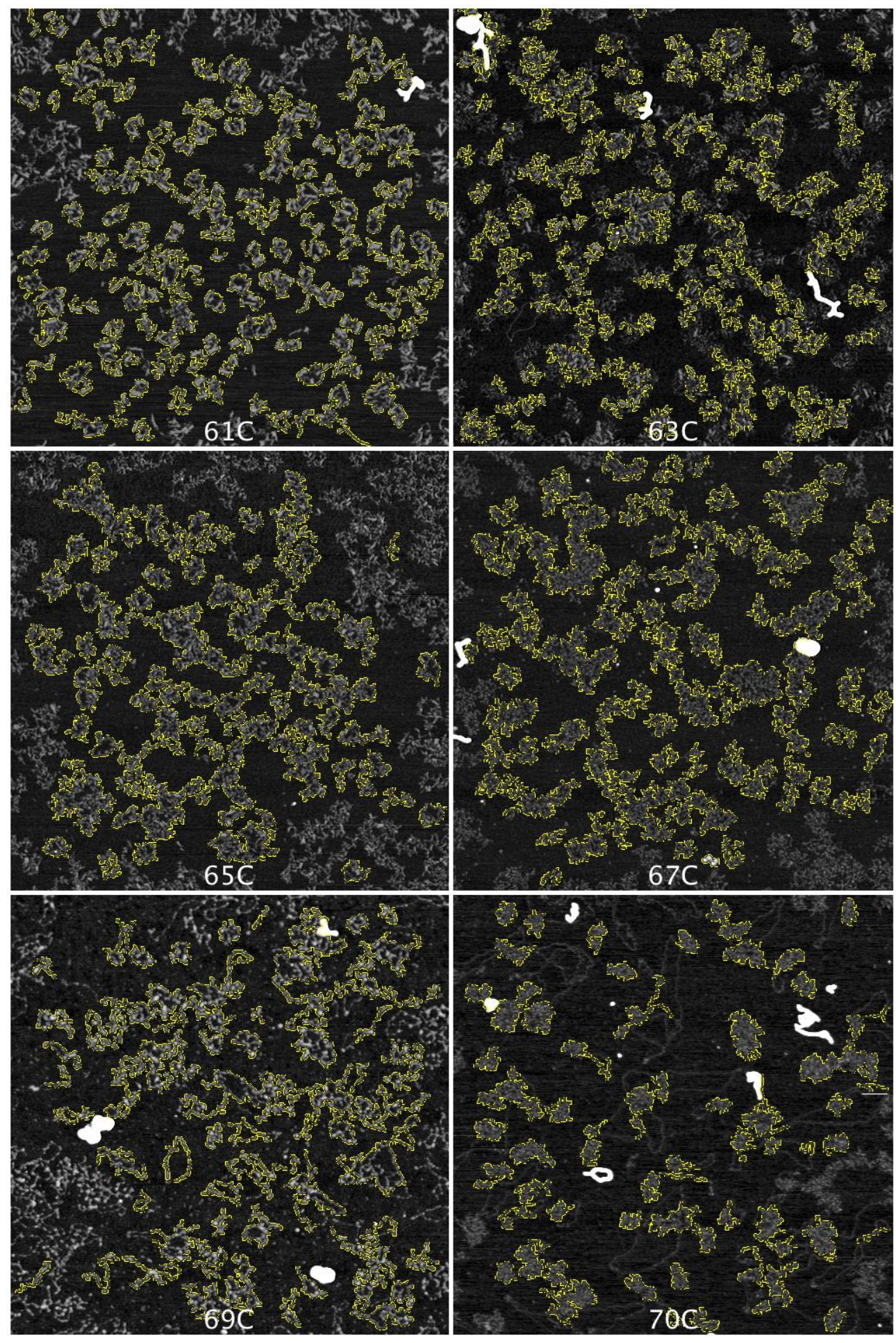

Figure S2: Detected particles (perimeter in yellow) during DNA tall rectangle folding while cooling. Data associated to Figure 2 and 3 in the Main Text. All images are $3 \times 3 \mu \mathrm{m}^{2}$. 


\section{Detected particles during melting}

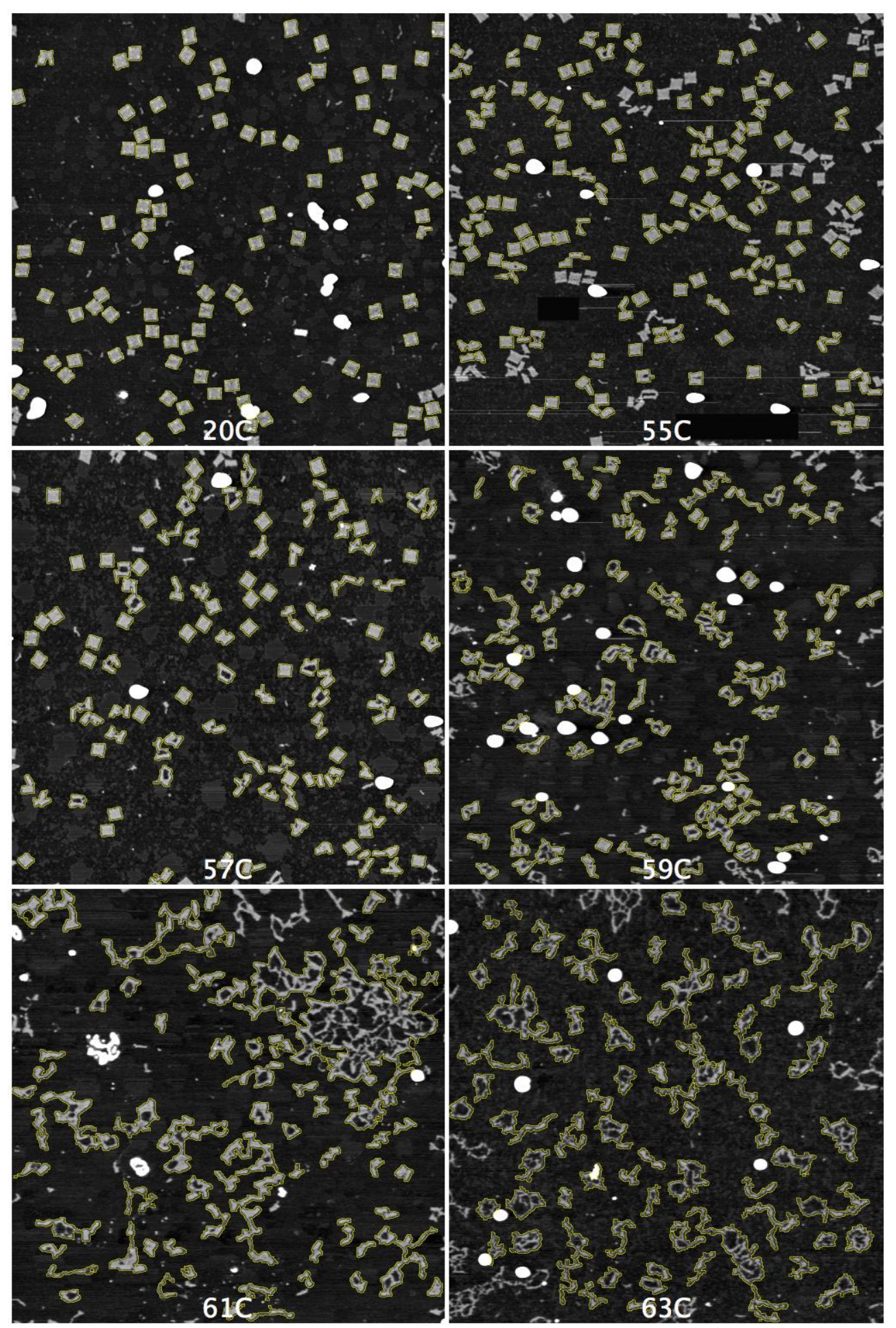

Figure S3: Detected particles (perimeter in yellow) during DNA tall rectangle melting while heating. Data associated to Figure 4 in the Main Text. All images are $3 \times 3 \mu \mathrm{m}^{2}$. 


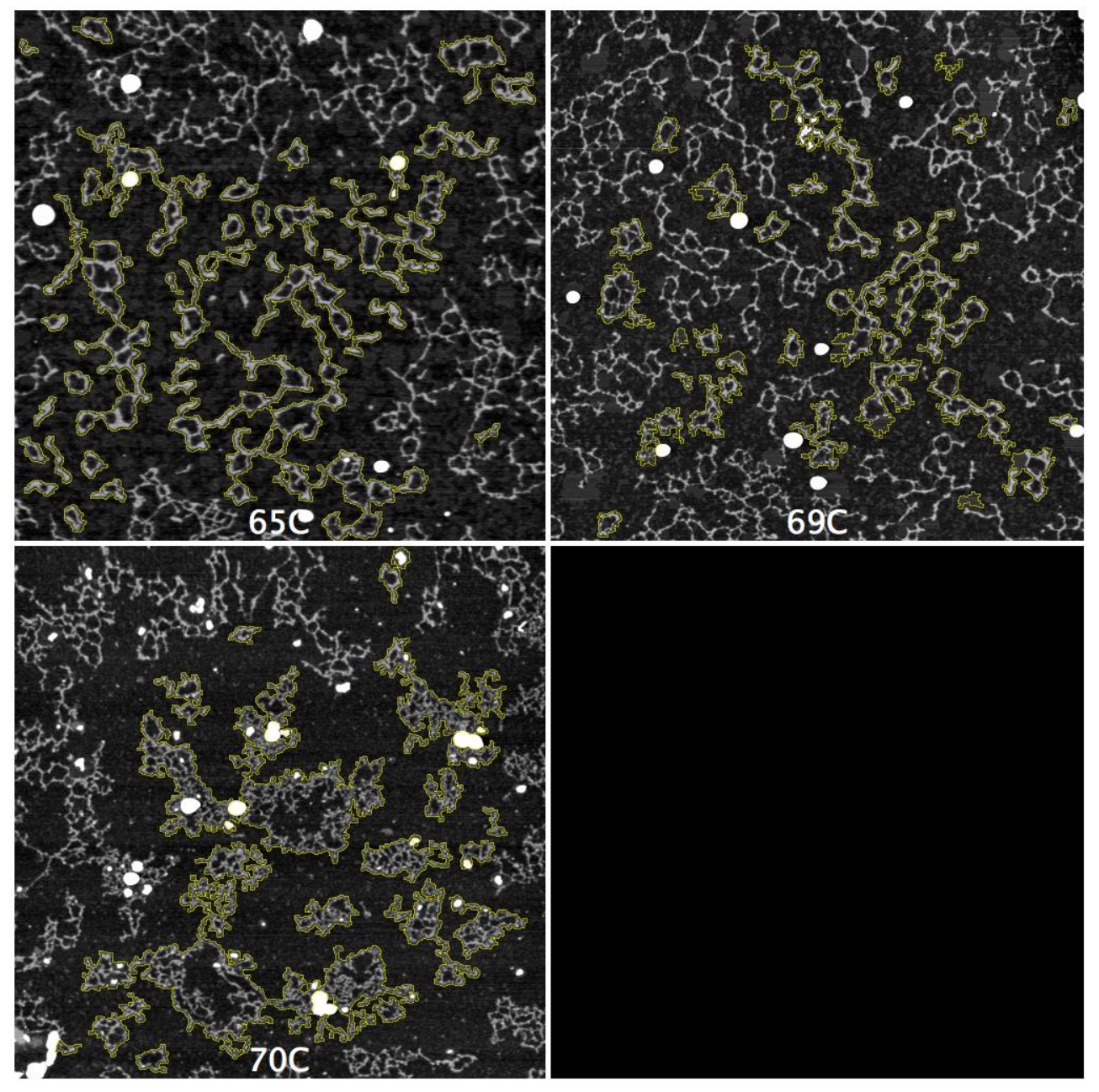

Figure S4: Detected particles (perimeter in yellow) during DNA tall rectangle melting while heating. Data associated to Figure 4 in the Main Text. All images are $3 \times 3 \mu \mathrm{m}^{2}$. 


\section{Variability of tall rectangle size as measured by AFM in different days}
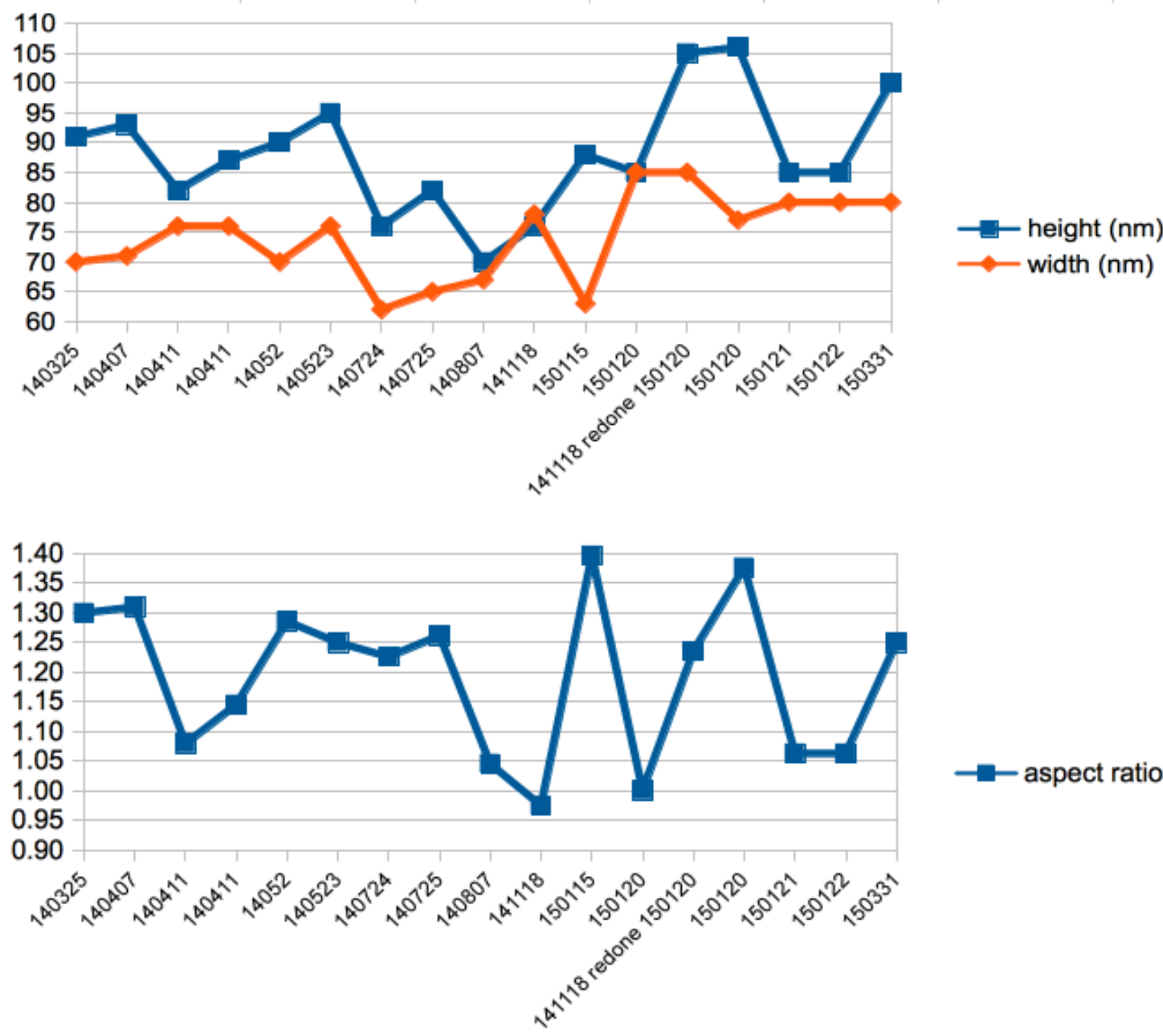

Figure S5: The size and aspect ratio of the tall rectangle imaged by AFM varies within experiments performed in different days. Top: Height and width of the tall rectangle. Bottom: Aspect ratio. 


\section{Reproducibility of AFM images during folding}

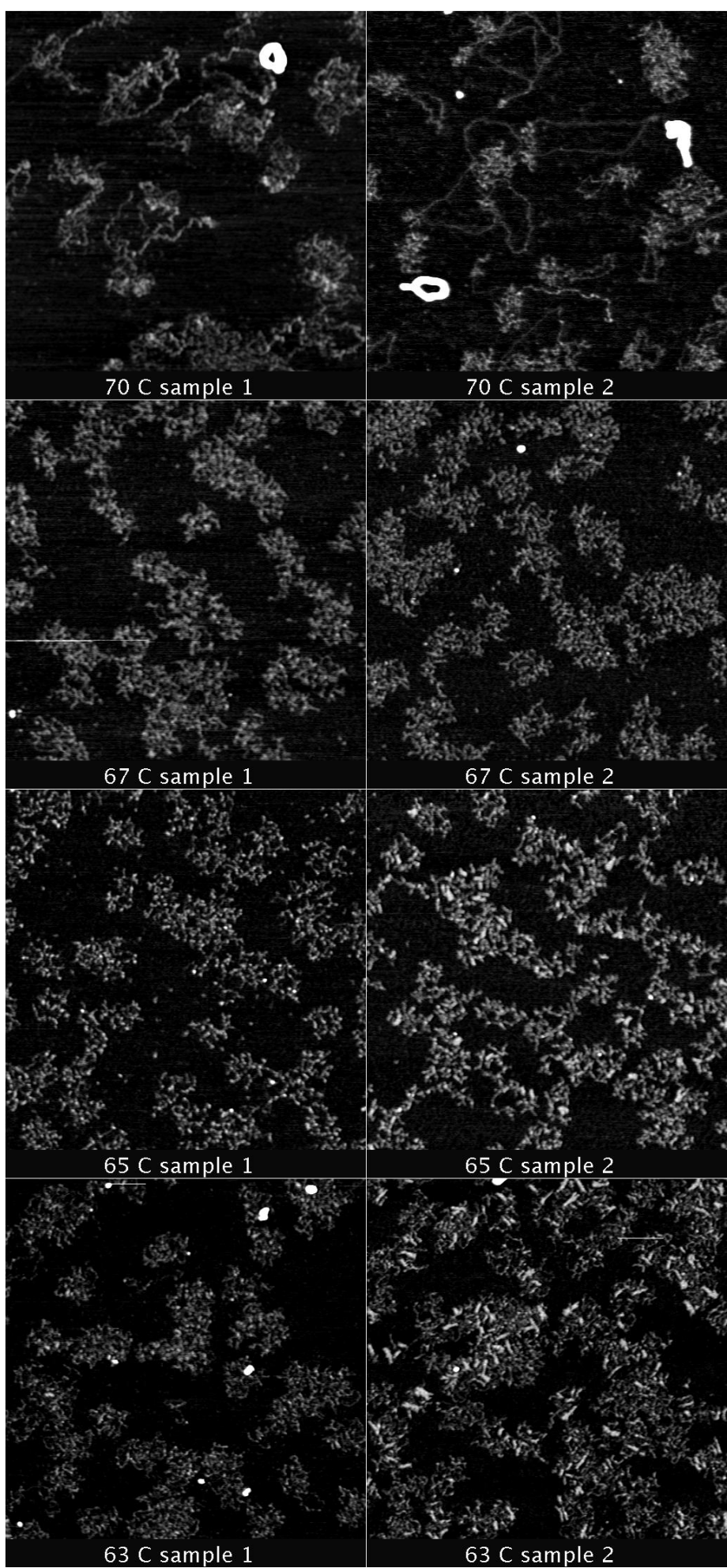

Figure S6: Reproducibility of deposition of foldamers on mica. AFM images during folding for two independent samples. Same conditions as in Figure 2 in the Main Text. All images are $1.5 \times 1.5 \mu \mathrm{m}^{2}$. 


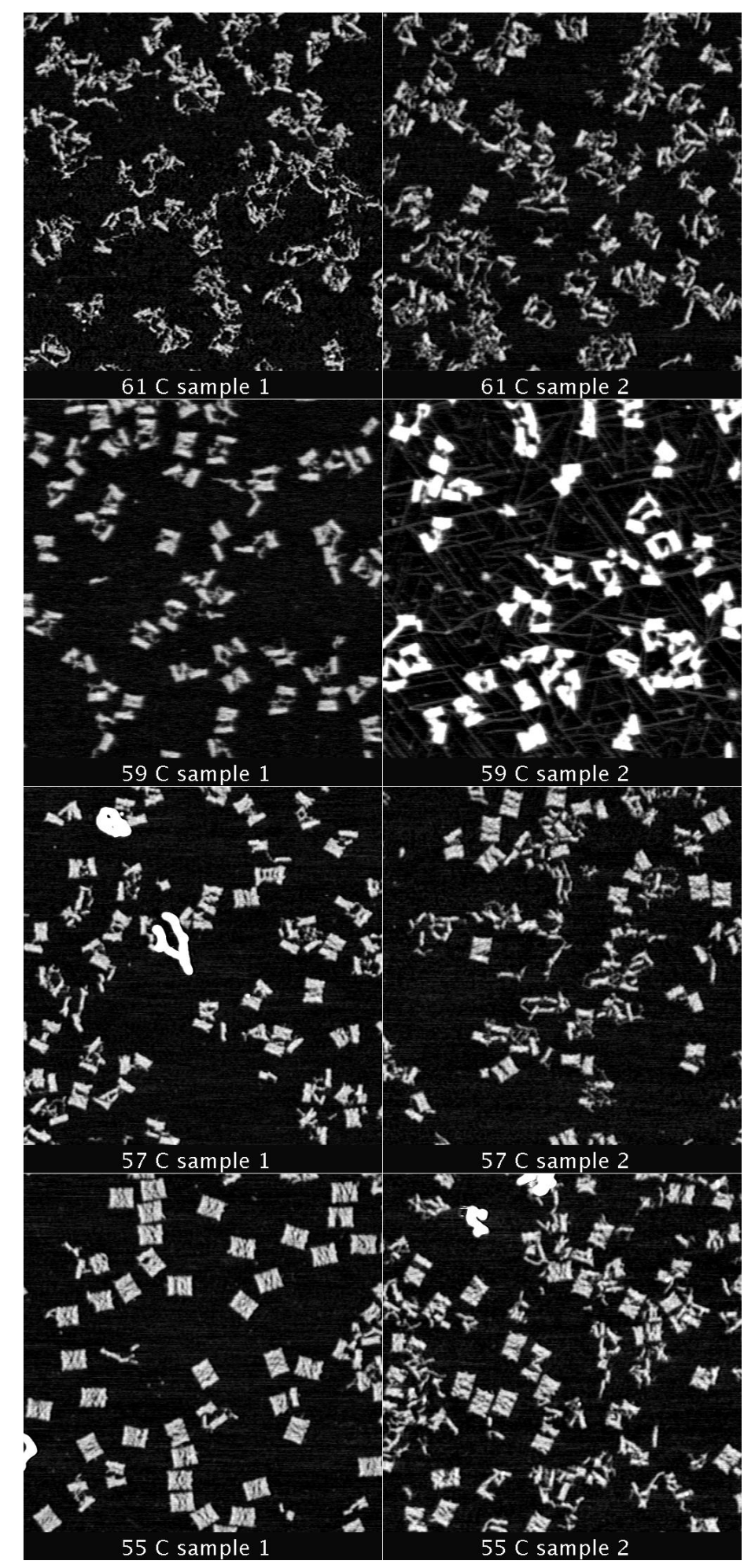

Figure S7: Reproducibility of deposition of foldamers on mica. AFM images during folding for two independent samples. Same conditions as in Figure 2 in the Main Text. All images are $1.5 \times 1.5 \mu \mathrm{m}^{2}$. 


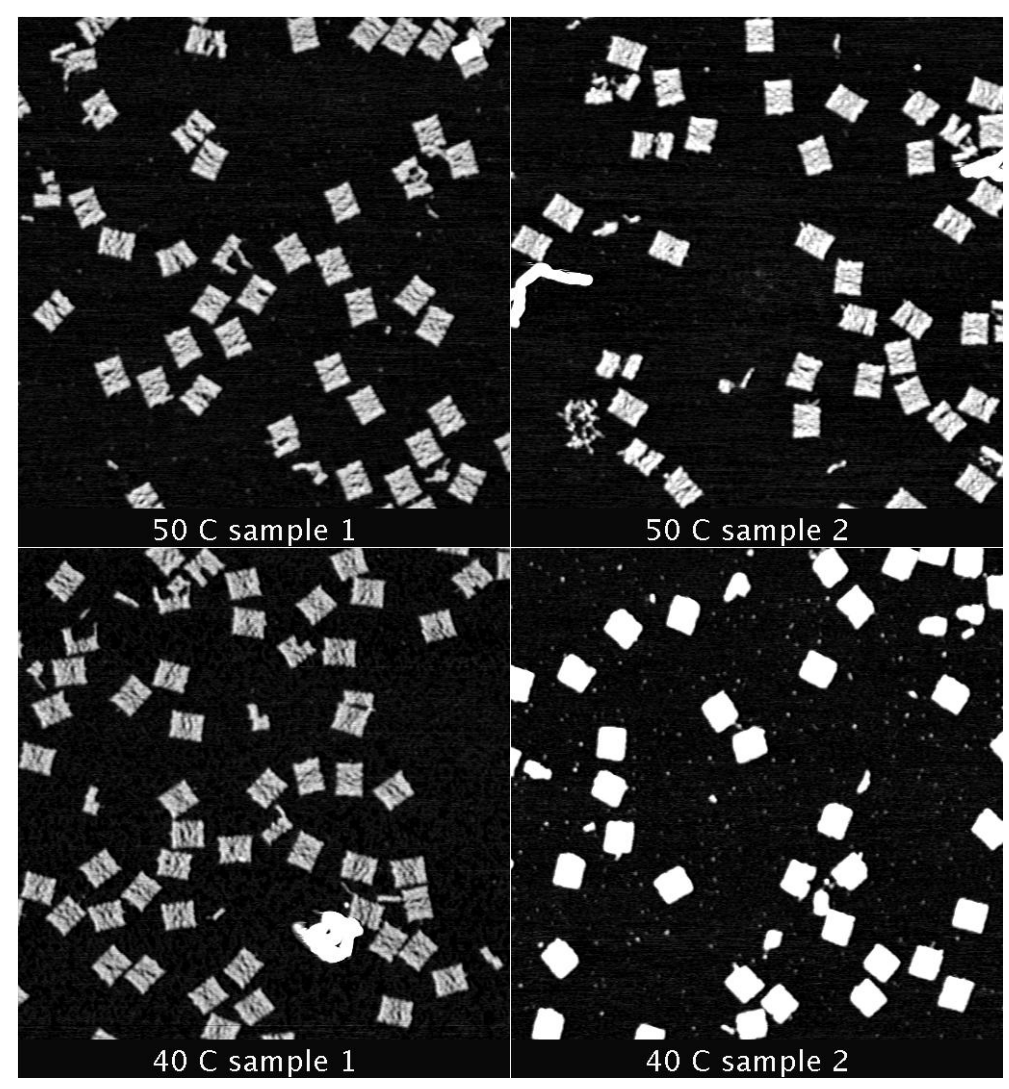

Figure S8: Reproducibility of deposition of foldamers on mica. AFM images during folding for two independent samples. Same conditions as in Figure 2 in the Main Text. All images are $1.5 \times 1.5 \mu \mathrm{m}^{2}$. 
Table S1: Frequency of fully-folded, broken rectangles and less folded structures (Other) during folding (Fold.) and melting (Melt.) counted manually in different mica samples (numbers) and different fields of view of the same mica sample (letters).

\begin{tabular}{ccccc}
\hline Sample & $\mathrm{T}\left({ }^{\circ} \mathrm{C}\right)$ & Fully folded & Broken rectangle & Other \\
\hline Fold. 1 & 55 & 0.2 & 0.2 & 0.6 \\
Fold. 2a & 55 & 0.7 & 0.3 & 0.0 \\
Fold. 2b & 55 & 0.8 & 0.2 & 0.0 \\
Fold. 1 & 57 & 0.1 & 0.3 & 0.6 \\
Fold. 2 & 57 & 0.2 & 0.6 & 0.3 \\
\hline Melt. 1 & 55 & 0.6 & 0.4 & 0.0 \\
Melt. 2 & 57 & 0.4 & 0.5 & 0.1 \\
\hline
\end{tabular}

\section{Effect of excess water rinsing on folded rectangle integrity}

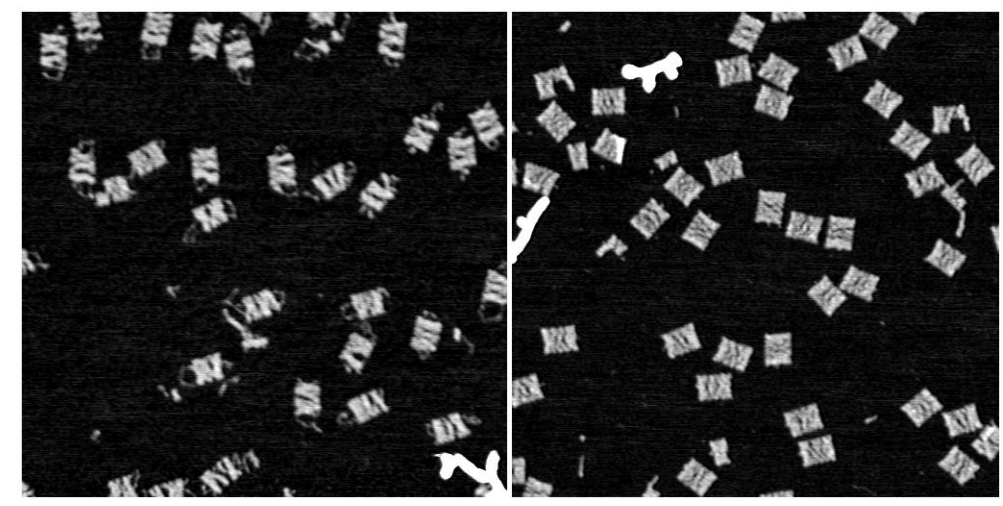

Figure S9: Folded tall rectangle washed with an excess of water (left) results in a characteristic structure where the top and bottom ends of the rectangle show loose loops. Right: control experiment. All images are $1.5 \times 1.5 \mu \mathrm{m}^{2}$. 


\section{Skipping a range of temperatures during folding}

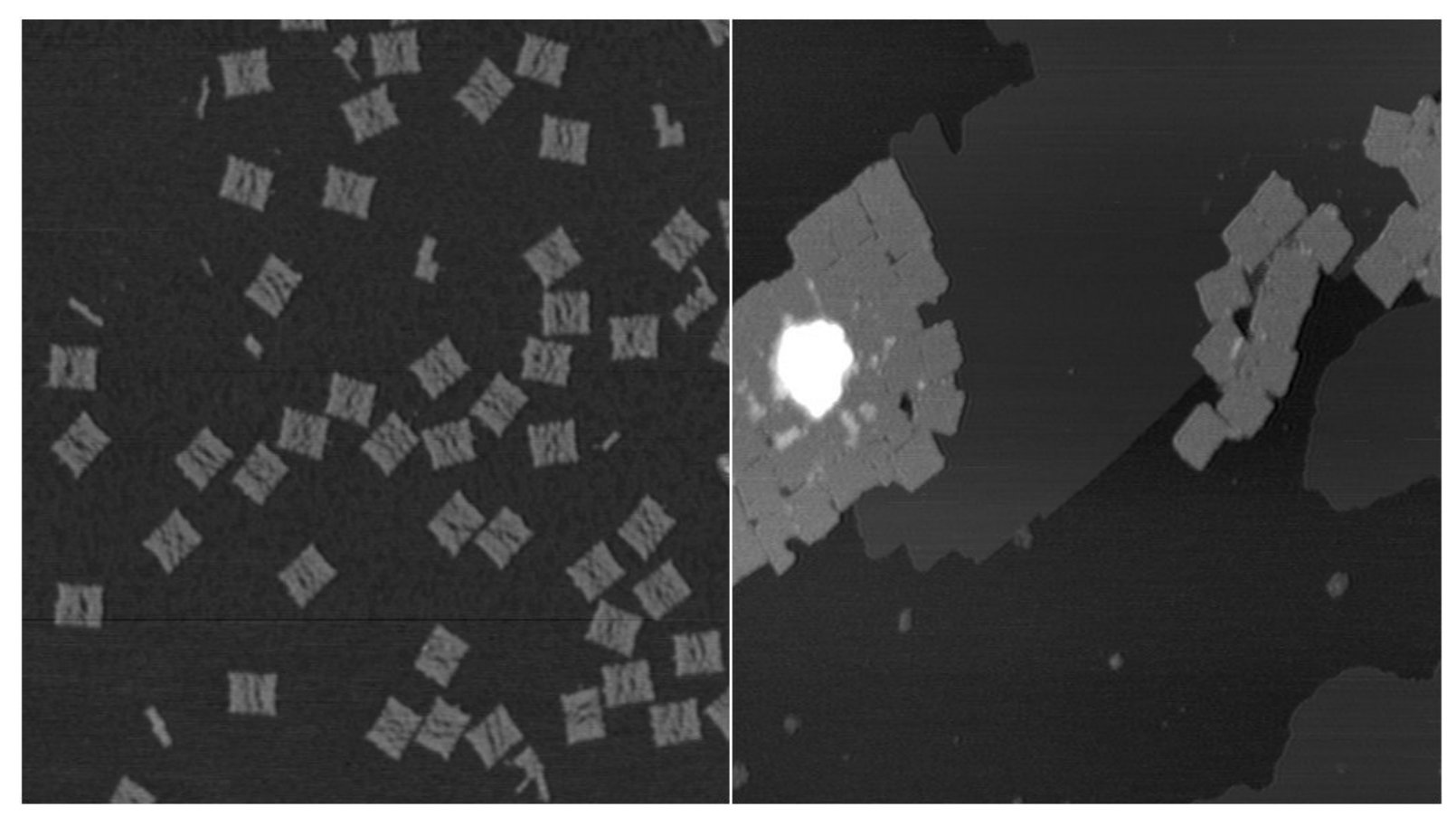

Figure S10: Comparison of AFM images at $40{ }^{\circ} \mathrm{C}$ for two different folding processes with $T_{\text {start }}=70{ }^{\circ} \mathrm{C}$, left, and $T_{\text {start }}=57^{\circ} \mathrm{C}$, right. The folding protocol consisted on a $10 \mathrm{~min}$ incubation at $95{ }^{\circ} \mathrm{C}$ followed by a sudden decrease until $T_{\text {start }}$ and then a temperature ramp of $-1{ }^{\circ} \mathrm{C} / 15 \mathrm{~min}$ until $40{ }^{\circ} \mathrm{C}$. Images are $1.5 \times 1.5 \mu \mathrm{m}^{2}$ with a height range of $0-1.3 \mathrm{~nm}$. Scaffold:staple 1:52 nM.

The results of Fig. 2 and 3 of the Main Text show that folding starts being visible at $63{ }^{\circ} \mathrm{C}$, suggesting that higher temperatures could be skipped for correct folding, as has been demonstrated by Sobczak et al 2012 (see the reference in the Main text). Fig. S10 demonstrates that we obtained correctly folded structures even when all the temperatures between 95 down to $57^{\circ} \mathrm{C}$ were skipped. 


\section{Quantitative analysis of AFM images}

\subsection{Quantitative analysis of melting}
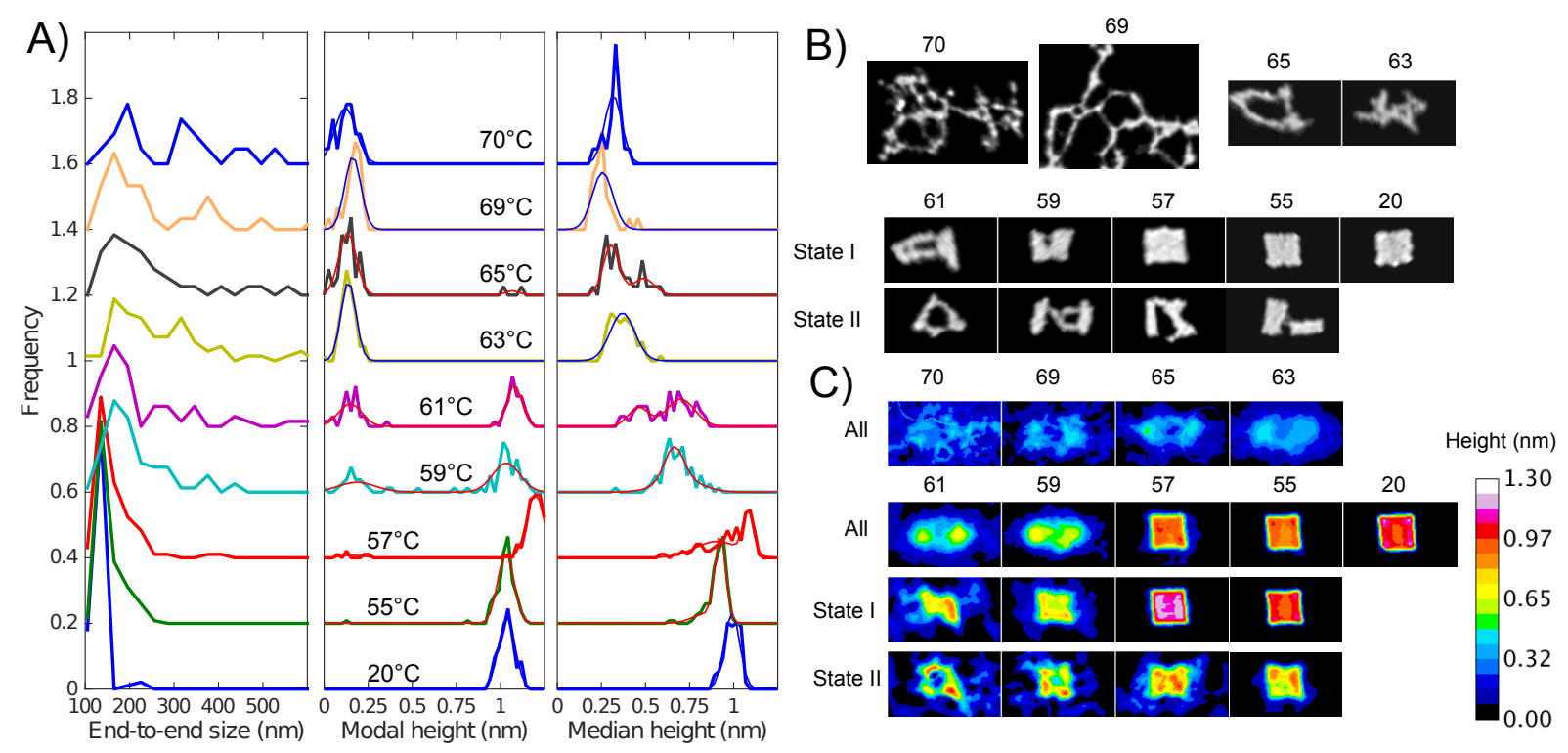

Figure S11: Population analysis of an ensemble of DNA origami tall rectangles melting while heating. Histograms of the end-to-end length, the modal height, and the median height of particles extracted from the whole field of view $\left(3 \times 3 \mu \mathrm{m}^{2}\right)$ of the images in Figure 4 in the Main Text (See also SI Figures S3 and S4), plots are offseted for clarity. Thin blue and red lines correspond to single and double gaussian fits, respectively. Between 40 and 100 particles were analyzed for each temperature. (B) AFM images of representative particles close to the maxima of the median height distribution and (C) average AFM images at each temperature. State I and II correspond to the two populations found in the range $61-55^{\circ} \mathrm{C}$. Images are $146 \times 88 \mathrm{~nm}^{2}$ except 70 and 69 that are larger, but drawn at the same scale. Heating rate $1^{\circ} \mathrm{C} / 10 \mathrm{~min}$. 


\subsection{Comparison of folding and melting}
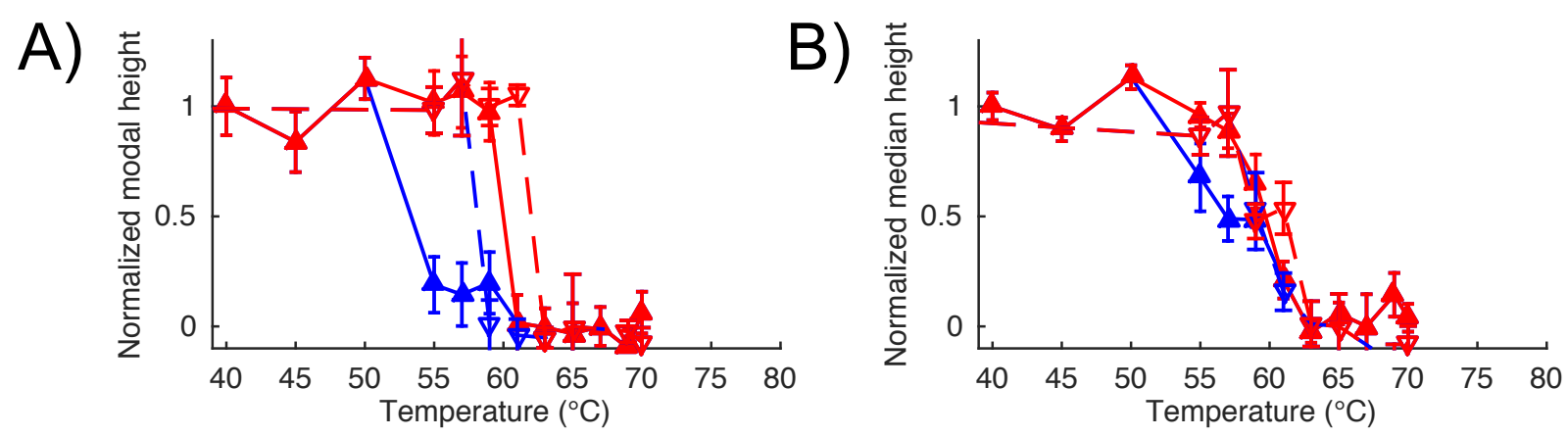

Figure S12: Normalized modal (A) and median height (B) for state I (blue) and state II (red) both for folding (upward triangles and plain lines) and unfolding (downward triangles and dashed lines) as a function of temperature in AFM experiments. Staples 52 nM, scaffold $1 \mathrm{nM}$. 


\section{Dependence of the melting temperature on the sta- ple concentration}

\subsection{Effect of staple concentration on nucleation site concentra- tion}

Let's consider the reaction of staple $i j$, called S, with the scaffold, T. Let's consider that the two sites are identical and call $\mathrm{C}$ the complex of $\mathrm{T}$ bound to one binding site of the staple, D the complex bound to two staples $\mathrm{S}$, and $\mathrm{O}$ the complex bound to one staple at its two sites. In the absence of cooperativity we can write

$$
\begin{aligned}
\mathrm{S}+\mathrm{T} & \stackrel{K_{1}}{\rightleftharpoons} \mathrm{C} \\
\mathrm{C}+\mathrm{S} & \stackrel{K_{1}}{\rightleftharpoons} \mathrm{D} \\
\mathrm{C} & \stackrel{K_{2}}{\rightleftharpoons} \mathrm{O},
\end{aligned}
$$

where $K_{i}$ is the corresponding equilibrium constant. We note in italics the equilibrium concentration of each species and write the law of mass action and the conservation of mass

$$
\begin{aligned}
K_{1} & =\frac{C}{S T} \\
K_{1} & =\frac{D}{S C} \\
K_{2} & =\bar{C} \\
T_{0} & =T+C+D+O \\
S_{0} & =S+C+2 D+O,
\end{aligned}
$$

where the index 0 indicates total concentration. We can rewrite these equations as

$$
\begin{aligned}
K_{1} & =\frac{C}{\left(S_{0}-C-2 D-O\right)\left(T_{0}-C-D-O\right)} \\
K_{1} & =\frac{D}{\left(S_{0}-C-2 D-O\right) C} \\
K_{2} & =\frac{O}{C} .
\end{aligned}
$$

If the staple concentration is in excess compared with the scaffold, $S_{0} \gg C+D+O$, we obtain

$$
\begin{aligned}
C & =\frac{K_{1} S_{0} T_{0}}{1+K_{1} S_{0}+K_{1} K_{2} S_{0}+\left(K_{1} S_{0}\right)^{2}} \\
D & =\frac{\left(K_{1} S_{0}\right)^{2} T_{0}}{1+K_{1} S_{0}+K_{1} K_{2} S_{0}+\left(K_{1} S_{0}\right)^{2}} \\
O & =\frac{K_{1} K_{2} S_{0} T_{0}}{1+K_{1} S_{0}+K_{1} K_{2} S_{0}+\left(K_{1} S_{0}\right)^{2}} .
\end{aligned}
$$


The question is whether increasing $S_{0}$ increases or decreases the equilibrium concentration of $\mathrm{O}$, the folded state of the scaffold. To answer it, we derive $O$ in respect to $S_{0}$ and find

$$
\frac{\mathrm{d} O}{\mathrm{~d} S_{0}}>0 \quad \text { if } \quad K_{1} S_{0}<1
$$

Moreover we have that

$$
\begin{array}{lll}
O>C & \text { if } & K_{2}>1 \\
C>D & \text { if } & K_{1} S_{0}<1 \\
O>D & \text { if } & K_{1} S_{0}<K_{2}
\end{array}
$$

\subsection{Estimating the change in $T_{m}$}

Let's consider now equilibrium 1 alone. With an excess of staples, $S_{0}>T_{0}$, the equilibrium constant is

$$
K_{1}(T) \frac{T_{0}}{\mathscr{C}^{0}}=\frac{\xi}{(1-\xi)\left(\frac{S_{0}}{T_{0}}-\xi\right)}
$$

where $\xi$ is the extent of reaction, defined between 0 and 1 , and $\mathscr{C}^{0}=1 \mathrm{M}$ is the reference concentration such that the equilibrium constant is adimensional. The melting temperature $T_{m}$ is defined when $\xi=\frac{1}{2}$. Thus,

$$
K\left(T_{m}\right)\left(\frac{S_{0}}{\mathscr{C}^{0}}-\frac{T_{0}}{2 \mathscr{C}^{0}}\right)=1
$$

we remind that

$$
R T \ln (K(T))=\Delta_{r} G^{\circ}(T)=\Delta_{r} H^{\circ}-T \Delta_{r} S^{\circ}
$$

where the last equality expresses the Ellingham approximation where $\Delta_{r} H^{\circ}$ and $\Delta_{r} S^{\circ}$ are supposed independent of temperature. Combining (20) and (21) we get

$$
T_{m}=\frac{\Delta_{r} H^{\circ}}{\Delta_{r} S^{\circ}+R \ln \left(\frac{S_{0}}{\mathscr{C}^{0}}-\frac{T_{0}}{2 \mathscr{C}^{0}}\right)} .
$$

In our experiments, the scaffold concentration $T_{0}$ remains constant while the staples concentration $S_{0}$, changes. To estimate the change in $T_{m}$ as a function of $S_{0}$ we can write

$$
\frac{T_{m}\left(S_{0,1}\right)}{T_{m}\left(S_{0,2}\right)}=\frac{\Delta_{r} S^{\circ}+R \ln \left(\frac{S_{0,2}}{\mathscr{C}^{0}}-\frac{T_{0}}{2 \mathscr{C}^{0}}\right)}{\Delta_{r} S^{\circ}+R \ln \left(\frac{S_{0,1}}{\mathscr{C}^{0}}-\frac{T_{0}}{2 \mathscr{C}^{0}}\right)}
$$

We will now proceed to an order of magnitude estimation of the expected change in $T_{m}$ when $S_{0}$ changes. A staple has typically $30 \mathrm{nt}$, half hybridizing on one side of the scaffold and another half on a different side. It is difficult to know which of the two reactions should one consider, the hybridization of a 15 mer or of a 30 mer. We will here consider the second case, which is the less favorable for our purpose. The nearest neighbor algorithm of Dinamelt yields $\Delta_{r} S^{\circ}=-3000 \mathrm{JK}^{-1} \mathrm{~mol}^{-1}$ for a $30 \mathrm{mer}$. In the standard conditions 
of the Main text $S_{0,1}=52 \mathrm{nM}$ and $T_{0}=1 \mathrm{nM}$. Let's see what happens when the staple concentration of the border of the origami is brought to $S_{0,2}=1 \mathrm{nM}$,

$$
\frac{T_{m}\left(S_{0,1}=52 n M\right)}{T_{m}\left(S_{0,2}=1 n M\right)}=1.01
$$

if we take as a typical melting temperature of border staples $T_{m}\left(S_{0,1}=52 \mathrm{nM}\right)=61{ }^{\circ} \mathrm{C}$, we have that

$$
T_{m}\left(S_{0,1}=52 \mathrm{nM}\right)-T_{m}\left(S_{0,2}=1 \mathrm{nM}\right) \approx 4{ }^{\circ} \mathrm{C}
$$

so the new $T_{m}$ for the border staples would be $57{ }^{\circ} \mathrm{C}$, which is not far from the $55^{\circ} \mathrm{C}$ measured experimentally. Let's now see what happens when the staple concentration of the center of the origami is brought to $S_{0,2}=670 \mathrm{nM}$, which is the highest concentration that is experimentally possible.

$$
\frac{T_{m}\left(S_{0,1}=52 \mathrm{nM}\right)}{T_{m}\left(S_{0,2}=670 \mathrm{nM}\right)}=0.992
$$

if we take as a typical melting temperature of border staples $T_{m}\left(S_{0,1}=52 \mathrm{nM}\right)=55{ }^{\circ} \mathrm{C}$, we have that

$$
T_{m}\left(S_{0,1}=52 \mathrm{nM}\right)-T_{m}\left(S_{0,2}=670 \mathrm{nM}\right) \approx-2.5^{\circ} \mathrm{C}
$$

and the new melting temperature is expected to be $57.5^{\circ} \mathrm{C}$, not far from the measured $59{ }^{\circ} \mathrm{C}$. 


\section{Melting curves in bulk}

\subsection{Influence of the temperature rate}
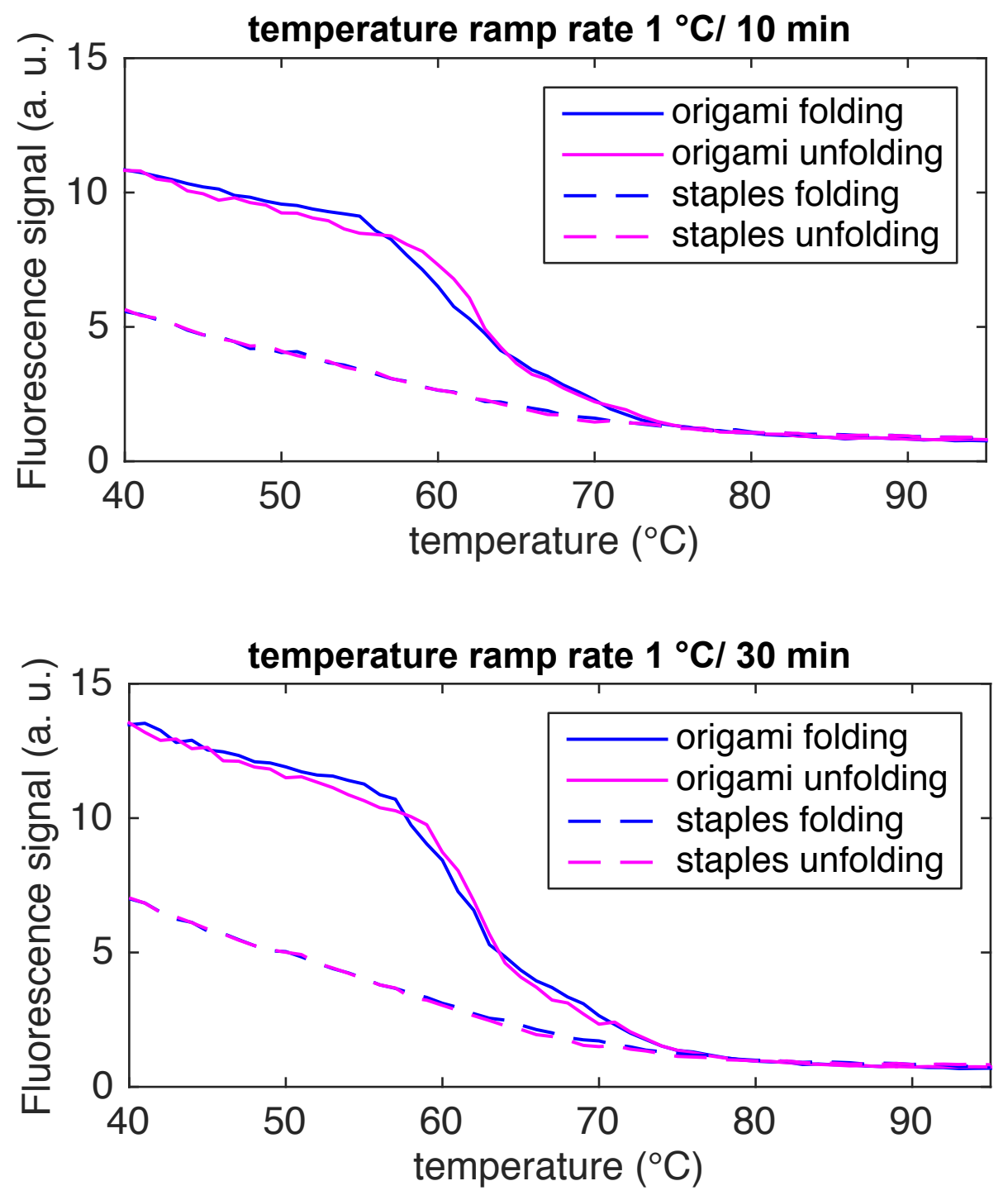

Figure S13: Raw fluorescence SybrGreen I signal for a 10:52 nM scaffold:staples and a 52 $\mathrm{nM}$ staples solution for cooling and heating at two different rates $1 / 10$ and $1 / 30{ }^{\circ} \mathrm{C} / \mathrm{min}$.

\subsection{Influence of relative staple concentration}

When the central staples were in excess and the edge staples almost stoechiometric (compared with the scaffold concentration) the folding curve in bulk clearly changed. The onset of folding was displaced to lower temperatures, the interval of temperatures during which folding was observed was reduced and the final fluorescence signal was also reduced. 


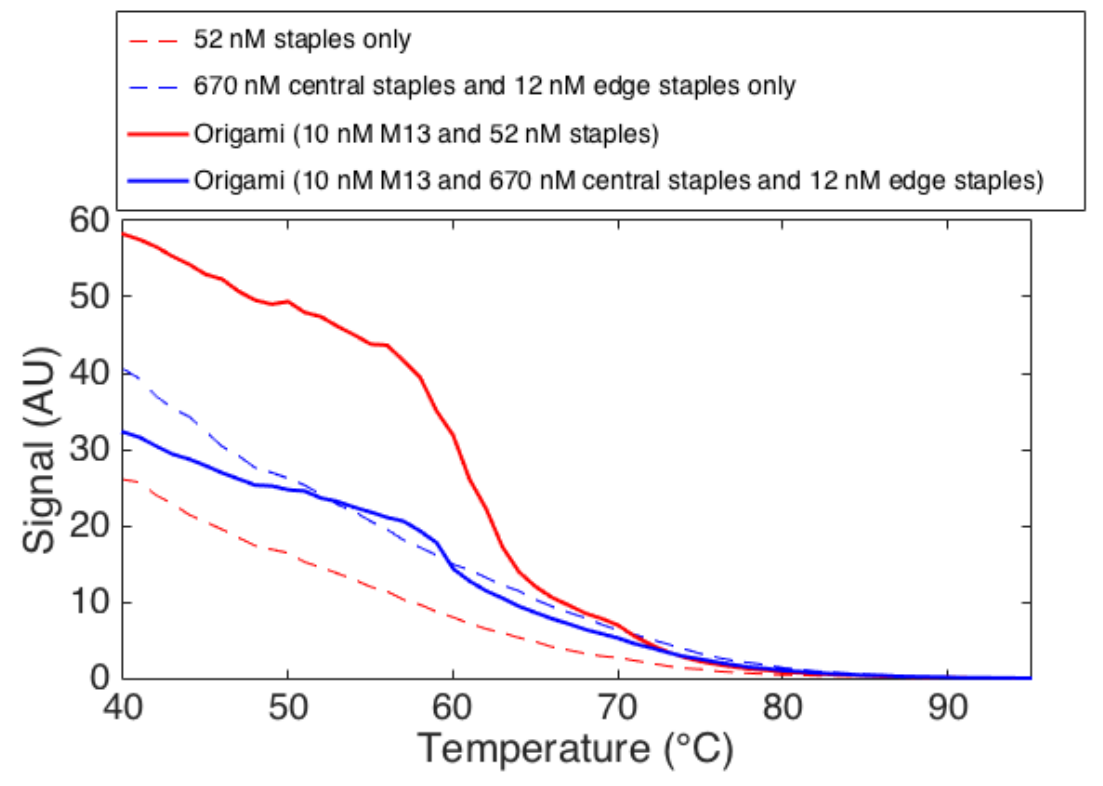

Figure S14: Raw fluorescence SybrGreen I signal for standard 52 nM staples (red) and 12 nM edge, $670 \mathrm{nM}$ central staples, with (solid) or without (dashed) $10 \mathrm{nM}$ M13 scaffold. All curves are correspond to cooling at $1 / 30^{\circ} \mathrm{C} / \mathrm{min}$. 


\section{Evaluation of the thermal effect of dipping mica on a solution of different temperature}

We consider a $3 \times 5 \times 0.1 \mathrm{~mm}^{3}$ piece of mica at temperature $T_{m}$ that is immersed in a $150 \mu \mathrm{L}$ water solution at temperature $T_{w}$. We look for the temperature change during such process. The specific heats of mica and water are, respectively, $c_{p, m}=0.88 \mathrm{Jg}^{-1} \mathrm{~K}^{-1}$ $c_{p, w}=4.19 \mathrm{Jg}^{-1} \mathrm{~K}^{-1}$ and their thermal conductivities $k_{m}=0.71 \mathrm{Wm}^{-1} \mathrm{~K}^{-1}$ and $k_{w}=$ $0.58 \mathrm{Wm}^{-1} \mathrm{~K}^{-1}$. At equilibrium we have

$$
\left(T_{f}-T_{m}\right) m_{m} c_{p, m}=\left(T_{w}-T_{f}\right) m_{w} c_{p, w}
$$

where $m_{i}$ is the mass of each substance. Reorganizing we have

$$
\left(T_{f}-T_{w}\right)=\left(T_{m}-T_{w}\right) \frac{m_{m} c_{p, m}}{m_{w} c_{p, w}+m_{m} c_{p, m}} .
$$

In the worst case, in our experiments we have $T_{w}=70^{\circ} \mathrm{C}$ and $T_{m}=20^{\circ} \mathrm{C}$. Otherwise, we have $m_{m}=4.5 \times 10^{-4} \mathrm{~g}$ (the density of mica being approximately $3 \mathrm{~g} / \mathrm{mL}$ ) and $m_{w}=1.5 \times 10^{-1} \mathrm{~g}$, thus

$$
\left(T_{w}-T_{f}\right)<0.3^{\circ} \mathrm{C}
$$

which is well below the $2^{\circ} \mathrm{C}$ precision of our method. Moreover the above calculation was made for an isolated system, while in our experiments the solution is in contact with a thermostat when mica is dipped.

Now we can calculate the time to thermal equilibrium as

$$
\tau=\frac{m_{m} c_{p, m} d}{k A}=3 \times 10^{-2} \mathrm{~s}
$$

which is also negligible. 


\section{Image segmentation procedure}

The file segmentation_procedure.zip file associated to the Main Text contains the following files:

- align_origami_particles_from_overlay.m

- convert_origami_csv_results_into_mat.m

- origami_32bit_find_particles_in_dir.ijm

- origami_32bit_find_particles_in_dir_test_thresholds.ijm

- origami_histograms_and_gauss_fit.m

- origami_image_registration.m

- results_property_name.mat

They should be executed as follows:

- Store your 32 bit AFM images after background correction by Gwyddion in a folder called WORK_FOLDER/tiff/. Each image should be named STRING_XXC_STRING.tif, where XX is the temperature in celsius.

- If you wish to optimize the thresholds value for binarizing tiff images, make a copy of the contents of WORK_FOLDER/tiff/ into WORK_COPY/tiff/. Run origami_32bit_find_particles_in_dir_test_thresholds.ijm in Fiji. Visually inspect images in WORK_COPY/overlays/ and decide which threshold best detects particles at each temperature and change file origami_32bit_find_particles_in_dir.ijm accordingly.

- Run origami_32bit_find_particles_in_dir.ijm in Fiji in the WORK_FOLDER folder. After running this macro generates four new folders: WORK_FOLDER/mask/ : contains (rawImage)*(binaryMask) and the residuals (rawImage)*(1- binaryMask); WORK_FOLDER/numbered_mask/: contains the mask binary image where pixels are set to the particle number; WORK_FOLDER/overlay/ : contains raw images with overlays of the detected particles; WORK_FOLDER/results/ : contains .csv data with parameters extracted from the detected particles.

- Open Matlab. Make sure that you have a copy of the file results_property_name.mat in your working folder. Then, run convert_origami_csv_results_into_mat.m and select WORK_FOLDER when prompted. The result is a mat file in the working folder, such as WORK_FOLDER/EXP_NAME. mat, containing particle analysis data.

- Run align_origami_particles_from_overlay.m and select the file WORK_FOLDER/EXP_NAME. mat when prompted. Three new folders are created: WORK_FOLDER/facebook_list_align/, containing pre-alligned images of single 
particles; WORK_FOLDER/aligned_avg/, containing average images of pre-alligned particles; and WORK_FOLDER/corr/ that may contain particle-particle correlation images.

- Select a a 32-bit tiff model image against which register all the other and store it in WORK_FOLDER/. Then, run origami_image_registration.m and select this model image when prompted. A new folder called WORK_FOLDER/facebook_registered is created, containing a list of registered images of individual particles at each temperature and an /avg folder containing averages of registered particles. This step may take several minutes.

- Run origami_histograms_and_gauss_fit.m and open the EXP_NAME.mat file when prompted. Three new folders are created: histo containing histograms of particle parameters, with and without gaussian fits; facebook_selected_median and facebook_selected_mode containing, respectively, registered particles close the the maxima of the median and mode histograms. Within these two folders an extra pathways folder contains instances of folding pathways showing most representative particles at each temperature, with subfolders state1 and state2 corresponding to state I and II in the Main Text. 